\title{
On the role of lagged exogenous variables and spatio-temporal correlations in improving the accuracy of solar forecasting methods
}

\author{
Athanassios Zagouras, Hugo T. C. Pedro, Carlos F. M. Coimbra* \\ Department of Mechanical and Aerospace Engineering, Jacobs School of Engineering \\ Center of Excellence in Renewable Energy Integration and Center of Energy Research \\ University of California San Diego, La Jolla, California, 92093, USA
}

\begin{abstract}
We propose and analyze a spatio-temporal correlation method to improve forecast performance of solar irradiance using gridded satellite-derived global horizontal irradiance (GHI) data. Forecast models are developed for seven locations in California to predict 1-hour averaged GHI 1, 2 and 3 hours ahead of time. The seven locations were chosen to represent a diverse set of maritime, mediterranean, arid and semi-arid micro-climates. Ground stations from the California Irrigation Management Information System were used to obtain solar irradiance time-series from the points of interest. In this method, firstly, we define areas with the highest correlated time-series between the satellite-derived data and the ground data. Secondly, we select satellite-derived data from these regions as exogenous variables to several forecast models (linear models, Artificial Neural Networks, Support Vector Regression) to predict $\mathrm{GHI}$ at the seven locations. The results show that using linear forecasting models and a genetic algorithm to optimize the selection of multiple time-lagged exogenous variables results in significant forecasting improvements over other benchmark models.
\end{abstract}

Keywords: solar forecasting, time-lagged correlation, gridded data, exogenous input data, genetic algorithm

\section{Introduction}

Large-scale utilization of solar energy for power generation requires advanced forecasting technologies to handle the variability of this weather-dependent resource. Several research groups have developed forecast algorithms and methodologies to predict ground irradiance at different forecasting horizons, ranging from minutes out to days ahead into the future [1].

The numerical tools that ingest relevant ground telemetry, remote sensing data and numerical weather prediction results typically include simple deterministic models with complex preprocessing steps, stochastic time series models such as AR, ARMA and ARIMA, machine learning tools such as Artificial Neural Networks, K-Nearest-Neighbors, Supported Vector Regression, and many other post-processing algorithms [1-4]. Most existing operational models use only local data and are limited to observe variations in the input variables in a single point in space, and are "blind" to the motion of weather systems across the earth's surface. A few recent studies employed spatially distributed data to cover small regions in space. Linij et al. [5] use data from a network of 80 rooftop sensors in Tucson, AZ, Yang et al. [6] use a network of 10 sensors in Singapore and Sözen et al. [7] train a neural network for 12 ground stations spread over Turkey.

In both cases the researchers reported substantially improvements to the forecast skill. The drawback for such approach is that, a network of ground sensors is costly to deploy and maintain due to required regular maintenance. When no such ground truth data is available, is it possible to find some useful approximations to irradiance measurements as Pelland et al. [8] have done by using spatially distributed irradiance forecast from the Canadian Meteorological Centre. Al-Alawi and Al-Hinai in [9] developed a neural network based forecasting model to predict the global radiation for remote locations in Oman, where ground-based measurements are not available.

${ }^{*}$ Corresponding author: ccoimbra@ucsd.edu (Carlos F. M. Coimbra) 
In a similar way to these authors, we explore in this work simple forecast models that include irradiance data from locations in the vicinity of the point of interest. Here gridded data comes, not from a numerical weather prediction, but from models that ingest satellite images to estimate the solar irradiance at the ground level. Such models have grown in accuracy in recent years (see for instance $[10,11]$ ). Moreover, such data will even be more accurate in the near future with GOES-R, the next generation of geosynchronous environmental satellites by NOAA, that will increase the frequency of full-disk imagery from 30 minutes to 5 minutes [12].

The objective of this paper is to introduce a forecasting approach that improves the prediction accuracy of ground measured irradiance data throughout the state of California through the use of exogenous satellite-derived variables. Two main aspects are explored by the proposed methodology. First is to investigate how exogenous input data obtained by difference source than a ground-based sensor at the point of interest affect the forecast of the measured observations. It is important to identify the seeking area around the vicinity of a ground location where a model can search for exogenous input data. Secondly, the time latency of both the endogenous and exogenous inputs plays a crucial role in the forecasting performance, as well as the optimization of selecting a number of exogenous variables from different time lags. Given a large dataset of gridded satellite data, we propose a time-lagged correlation analysis between the ground measured times series and the gridded data to define potential seeking regions based on the degree of correlation. We study different types of forecasting models including the persistent model, Artificial Neural Networks (ANN), Support Vector Regression (SVR), linear models using a variety of time-lagged input data and a linear model optimized by Genetic Algorithms (GA). The latter provided the best forecast accuracy for forecast horizons up to 3 hours ahead, with respect to any ground location and evaluated on different periodic partitions of an independent testing subset.

The remainder of the paper is organized in four parts: in Section $\S 2$, the case study will be presented. Section $\S 3$ is devoted to a description of the time-lagged correlation analysis and the developed models. Section $\$ 4$ analyses the experimental results. The conclusion is reported in Section $\$ 5$.

\section{Case Study}

\subsection{CIMIS ground data}

Ground-based hourly irradiance data used in this study were obtained from 7 weather stations operated by California Irrigation Management Information System (CIMIS). A network of 120 automated weather stations throughout the entire state of California constitute the CIMIS program managed by the Office of Water Use Efficiency (OWUE), California Department of Water Resources (DWR). Aiming to assist in the efficient management of irrigation water resources, DWR and the University of California, Davis established the CIMIS program in 1982. The CIMIS weather stations are located at key agricultural and municipal sites to either measure various meteorological data on an hourly basis or calculate them from measure values. The weather stations are equipped to measure data such as solar radiation, air and soil temperature, relative humidity, precipitation, and wind speed and wind direction. In the present study, we selected seven weather stations located at distinct sites in California, from the north to the south as well as at coastal and inland sites (Fig 1). The wide range of stations was based on the estimated solar variability that each location experiences and their geographic proximity to regions where antipodal climatic conditions occur. For instance, San Diego is exposed to the marine layer of the Pacific ocean which substantially affects solar variability, whereas Merced, located in the Central Valley of California and has hot, dry, cloudless summers and notably low solar variability.

Table .2 summarizes geographical information for the sites used. Solar radiation data were obtained for a 45month period from January 2009 to September 2012. The total solar radiation that reaches each weather station is measured by a LI200S Li-Cor photovoltaic pyranometer. The 60 readings per minute are then averaged to hourly data and published on the CMIS portal. The error accuracy of the Li-Cor pyranometer is within $\pm 5 \%$ under natural sunlight conditions.

The ground data were split into training, validation and testing sets, respectively. The training set was used for the time-lagged correlation calculations and the estimation of the model parameters, the validation set was used for the parameter optimization and the testing was applied for evaluating the generalization capacity over independent data. The three data sets were created with the following algorithm: the $1^{\text {st }}$ week of every month was assigned to the validation set, the $2^{\text {nd }}$ to testing and the remaining data were used for training. This resulted in a typical data partition 


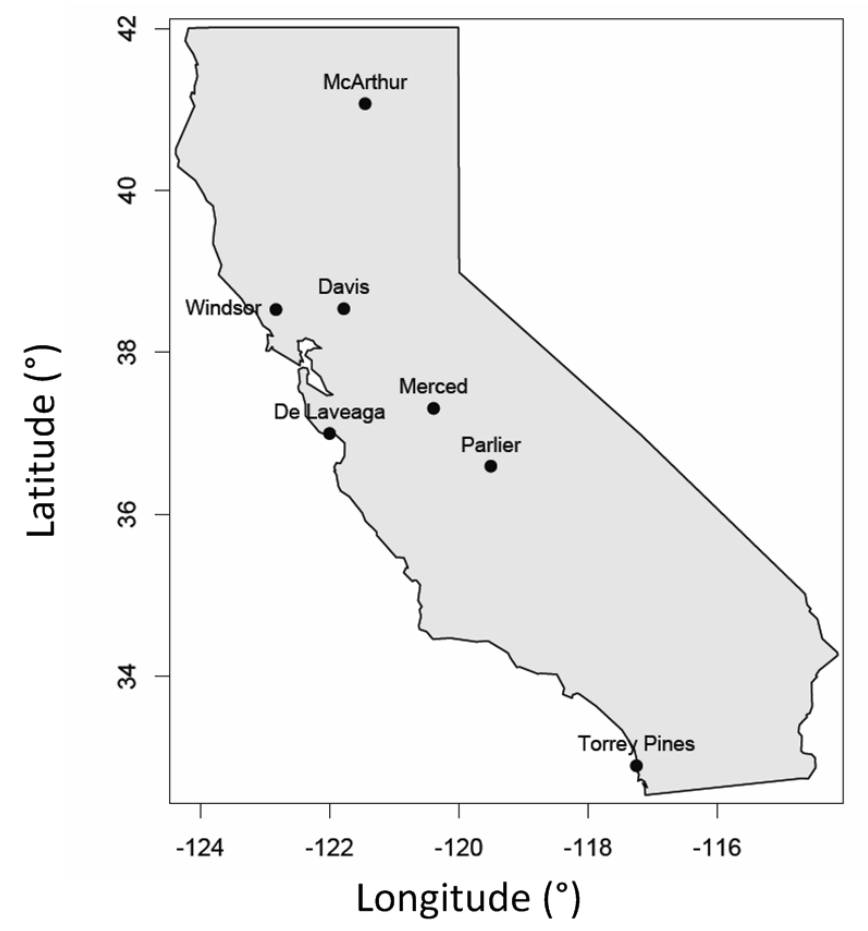

Figure 1: Map of California in gray with selected CIMIS weather stations marked in black and labeled.

with $50 \%$ for training and $25 \%$ for validation and testing, respectively. This splitting algorithm ensures that all the subsets share similar seasonal trends.

\subsection{Satellite-derived data}

In addition to the CIMIS data, we use gridded data values of GHI for the state of California provided by the SolarAnywhere ${ }^{\circledR}[13]$. The Enhanced Resolution dataset, for the same period as the CIMIS dataset, consists of satellite-derived GHI data on an approximately $0.01^{\circ}$ resolution grid both in latitude and longitude and a 30 min time sampling rate. SolarAnywhere ${ }^{\circledR}$ global and direct irradiance values are derived from the SUNY semi-empirical satellite-to-irradiance model, which relies on imagery collected from the GOES-W and GOES-E satellites in conjunction with other meteorological data sources $[14,15]$. A number of studies validates the accuracy of SUNY model at several individual locations with a typical error of the derived global and direct irradiance estimates correspond to 5\% and $10 \%$, respectively [14-16]. Particularly for the GHI data in the state of California, Nottrott and Kleissl [10] have demonstrated that SUNY model estimates GHI with an average mean bias error of 5\%, which is comparable with that of ground-based measurements.

In order to reduce the large number of grid nodes we applied a median filter to all the non-overlapping windows consisted of $5 \times 5$ nodes. The use of the median function is based on the consideration that adjacent nodes in the grid should have relatively smooth deviation of GHI values. Thus, every $25 \mathrm{~km}^{2}$ region (i.e. $5 \times 5$ grid) is represented by the median GHI value of the node values in the window. As the window slides over the entire image, the result from the median filter is assigned to a new grid, which is composed of the coordinates of the central node in each window. In order to match the 30-minute satellite-derived GHI with the 1-hour ground data we averaged the former dataset over 1 hour intervals $\left( \pm 30\right.$-min). Finally, the preprocessed SolarAnywhere $e^{\circledR}$ dataset, that shares common time stamps with the CIMIS dataset (Jan 2009 - Sept 2012), was also split into the three subsets described above.

\subsection{Clear-sky model and clear-sky index}

The clear-sky models (CSM) are employed by several applications in the solar engineering field to compute the expected ground irradiance for sky conditions at any location and time. In recent past a number of CSM has been 
presented which are associated with a variety of different climatic input parameters [17, 18]. The normalization of satellite data by the expected clear sky values constitutes an important prepossessing task where the normalized irradiance becomes independent from factors such as the geographic location, the time of year and the variability associated with deterministic solar diurnal cycle. In this study we employed the CSM proposed by Ineichen and Perez (2002) [19] which requires as the only atmospheric input the Linke turbidity factor at an airmass of 2. In contrast to other multi-parameter CSM in the literature, the selection of this model lies in the availability of broadband input parameters and the demonstrated good performance in the state of California [20]. Current research on the accuracy of diverse CSM has shown that the particular model is among the four best-performing models out of 54 CSM in total [21]. The CSM values, $G H I_{c s}$, were calculated for all the nodes of the reconstructed grid domain and the hourly averaged time stamps as described in the section $\S 2.2$. The clear sky index $k_{\text {exo/endo }}$, defines the normalized $G H I_{\text {exo/endo }}$ time series, which is calculated as:

$$
k_{\text {exo } / \text { endo }}(t)=\frac{G H I_{\text {exo } / \text { endo }}(t)}{G H I_{c s}(t)},
$$

where the subscripts exo/endo indicate either the satellite-derived data (the exogenous variables) or the ground measured GHI at the CIMIS stations (the endogenous variables). Both the $k_{\text {exo/endo }}$ time series values vary between 0 and 1 , representing fully overcast sky or completely clear conditions, respectively.

The datasets of temporal vectors $G H I_{\text {exo/endo }}$ and $k_{\text {exo/endo }}$ are created for the three time subsets described in the section $\$ 2.1$. They consist of $N$ time series vectors of the irradiance features (i.e., $G H I_{\text {exo/endo }}$ or $K_{\text {exo/endo }}$ ) for daytime periods. The number of vectors, $N=16625$, results from the total number of nodes of the reconstructed $\sim 5 \mathrm{~km} \times 5$ $\mathrm{km}$ grid and correspond only to land regions of California.

\section{Forecasting framework}

Using the datasets explained above we address a simple question: having access to gridded irradiance data (measured or modeled as in this case) can these data be used to improve irradiance forecast for a given location within the gridded data domain and how can this be done? The answer to the first part of the question is trivial: spatially distributed irradiance data should always improve the forecast accuracy. Cloud advection, for instance, cannot be detected with irradiance data from a single location but can be easily observed in gridded data. Large improvements in the forecast accuracy should be expected when such information is available.

The second part of the question has multiple possible answers and it depends on the preferences of the researcher. In the remainder of this paper we will provided our approach and assess its performance.

In general terms the forecast models here proposed are given by:

$$
\begin{aligned}
\hat{k}(t+\delta)=f & \left(k_{\text {endo }}(t), k_{\text {endo }}(t-\Delta t), \cdots, k_{\text {endo }}(t-i \Delta t),\right. \\
& k_{\text {exo }, 1}(t), k_{\text {exo }, 1}(t-\Delta t), \cdots, k_{\text {exo }, 1}(t-i \Delta t), \\
& \cdots, \\
& \left.k_{\text {exo }, j}(t), k_{\text {exo }, j}(t-\Delta t), \cdots, k_{\text {exo }, j}(t-i \Delta t), \cdots\right)
\end{aligned}
$$

where $\hat{k}(t+\delta)$ is the forecasted value at time $t+\delta, k_{\text {endo }}$ is the clear-sky value at the location of interest, and $k_{\text {exo }}$ is the clear-sky irradiance from exogenous locations obtained from the gridded data. Models where developed to forecast horizons $\delta=1,2,3$ hours. The number of exogenous locations $k_{\text {exo,j }}$ is not defined beforehand nor is the number of time lags $t-i \Delta t$. The approximation function $f$ can take various forms depending on the forecast model used. In this work we will explore the following tools: linear models, Artificial Neural Networks and Support Vector Regression. The dependent variable in these models is the clear-sky index and not the irradiance, given that contrary to GHI, the $k$ time series is stationary. However, all the results and error analysis presented ahead will be in terms of irradiance, which can be obtained as $\hat{I}(t)=\hat{k}(t) \times I_{c s}(t)$.

The choice of exogenous locations and time-lagged variables to be included in Eq. 2 will be addressed bellow. The optimal set of exogenous variables and time-lagged data will be determined through a genetic algorithm. However, given that any point in the California dataset could be used as an exogenous input we propose a preprocessing technique to reduce the set of candidates to a more manageable number. This step is based on the correlation analysis between the ground data for the selected locations and the gridded GHI data. 

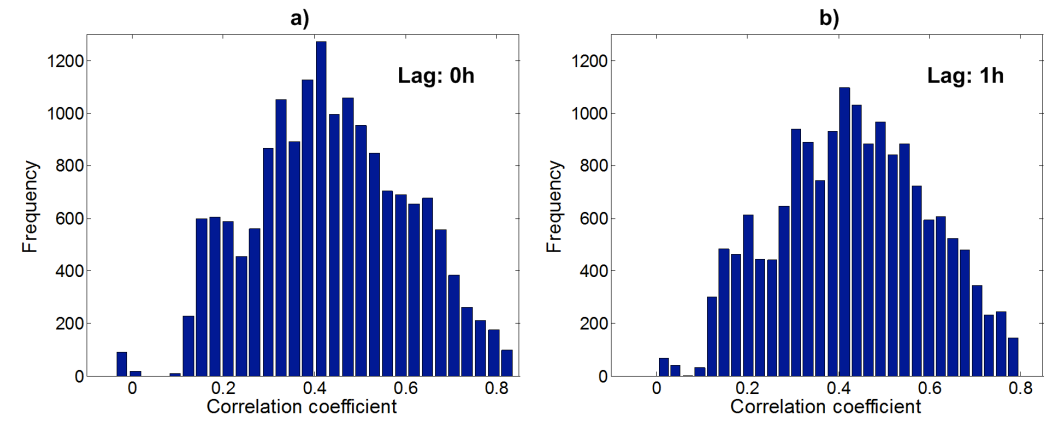

c)
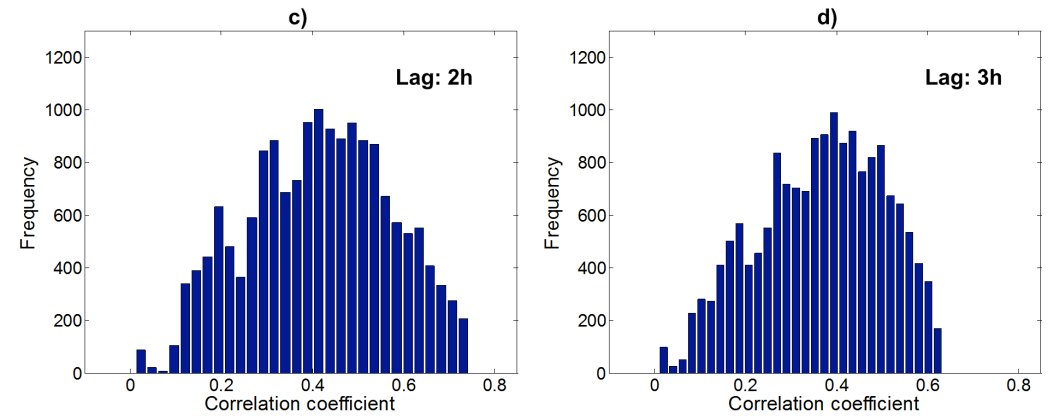

Figure 2: Frequency distribution of the Pearson $\rho$ correlation coefficients values between the CIMIS station at Merced and the time shifted satellitederived $K$ time series at CA by a) $0 \mathrm{~h}$, b) $1 \mathrm{~h}$, c) $2 \mathrm{~h}$, d) and $3 \mathrm{~h}$. The horizontal axis represents bins of correlation coefficients values, while the vertical axis refers to the number of map pixels occur at each bin.

\subsection{Time-lagged correlation analysis}

Time-lagged correlation analysis is a challenging approach of stochastic modeling to investigate the pairwise relationship between time shifted solar-irradiance time series, as a function of both the spatial distribution of the measurements sites and the time delays of the measured irradiance [22-24].

Before developing the forecast models in detail, it is necessary to examine two essential aspects. The first is to define the spatial area characterized by significant correlation between $k_{\text {exo }}$ and $k_{\text {endo }}$ time series for different locations. The exogenous inputs to Eq.2 will be selected from these areas. In general, it is expected that these areas will be formed by the grid nodes closest to the ground stations. The second aspect is to determine a criterion based on the correlation magnitude that will define these areas. In order to address these two issues, we performed a time-lagged correlation analysis between the $k_{\text {endo }}$ time series at each of the CIMIS locations with time-lagged $k_{\text {exo }}$ satellite-derived time series from the entire state of California. The Pearson correlation coefficient $\rho$ is defined as:

$$
\rho_{\tau, i}=\frac{\operatorname{cov}\left(k_{\text {endo }}(t), k_{\text {exo,i }}(t-\tau)\right)}{\sigma\left(k_{\text {endo }}(t)\right) \sigma\left(k_{\text {exo }, i}(t)\right)},
$$

with $i=1, \ldots, N$, where $\tau=\{0,1,2,3\}$ represents the hourly time lags applied to the $k_{\text {exo }}$ time series, and cov and $\sigma$ stand for covariance and standard deviation, respectively.

The "connection" between the $k$ time series of selected ground points of interest with a large satellite-derived auxiliary dataset of antecedent data can now be interpreted by a lag-dependent Pearson correlation coefficient map. All the time series used for the correlation analyses were obtained from the training subset. While we aim to short-term forecast horizons, the time lags were defined to vary between 0 and 3 hours.

A preprocessing step of the correlation analysis for each reference location requires to clean the CIMIS data from any missing values or invalid measurements and perform an overlapping match with the corresponding satellite data. Specifically, all the time stamps of an entire day are removed if a missing/invalid value during that day is detected. Although the number of overlapped data among the cases of different CIMIS locations is variable, they always exceed $90 \%$ of the initial dataset size. 
The group of grid points with the largest correlation to the reference point are used to form the area wherein exogenous time series can be selected as inputs to the forecast models. Once the correlation maps for the delay $\tau$ are computed and, given a threshold value $\rho_{\tau, \text { lim }}$ to define the set of nodes in the the search area $S$, the later is given as:

$$
\left.S_{\tau}\right|_{\rho_{\tau, \text { lim }}}=\left\{i: \rho_{\tau, i} \geq \rho_{\tau, l i m}\right\}, \tau=\{0,1,2,3\}
$$

and the set of exogenous variables associated to these indices is

$$
\left.K_{\tau}\right|_{\rho_{\tau, l i m}}=\left\{k_{\text {exo, }, i}: i \in S\right\}, \tau=\{0,1,2,3\}
$$

Figure 2 shows the distribution of correlation values for the different lags for Merced. The figure shows a number of grid points between 100 and 200 that have the highest correlation with the CIMIS ground time series for different time lags. The highest correlations decrease with increasing time lags. We therefore define the search areas for each CIMIS reference location as the regions of top-correlated pixels with respect to different time lags.

An example of the correlation map $\rho_{0}$ for Davis is illustrated in Fig. 3a. While an extensive area of well correlated locations (i.e. $\rho>0.5$ ) encloses the CIMIS station at Davis, we confine the search area to the 100 most correlated nodes, $\rho_{\text {lim }}=\rho_{100 t h}$. The search areas for the locations Davis, McArthur and Torrey Pines (San Diego) are shown with red in Fig. 3 (b-d), respectively. The blue areas indicate the nodes whose correlation with the ground data is smaller than the threshold. The shape of the search area is variable and depends on the threshold value and the location of reference. This evidence indicates the necessity of performing correlation analysis in order to accurately define the boundaries of the area of potential exogenous locations.
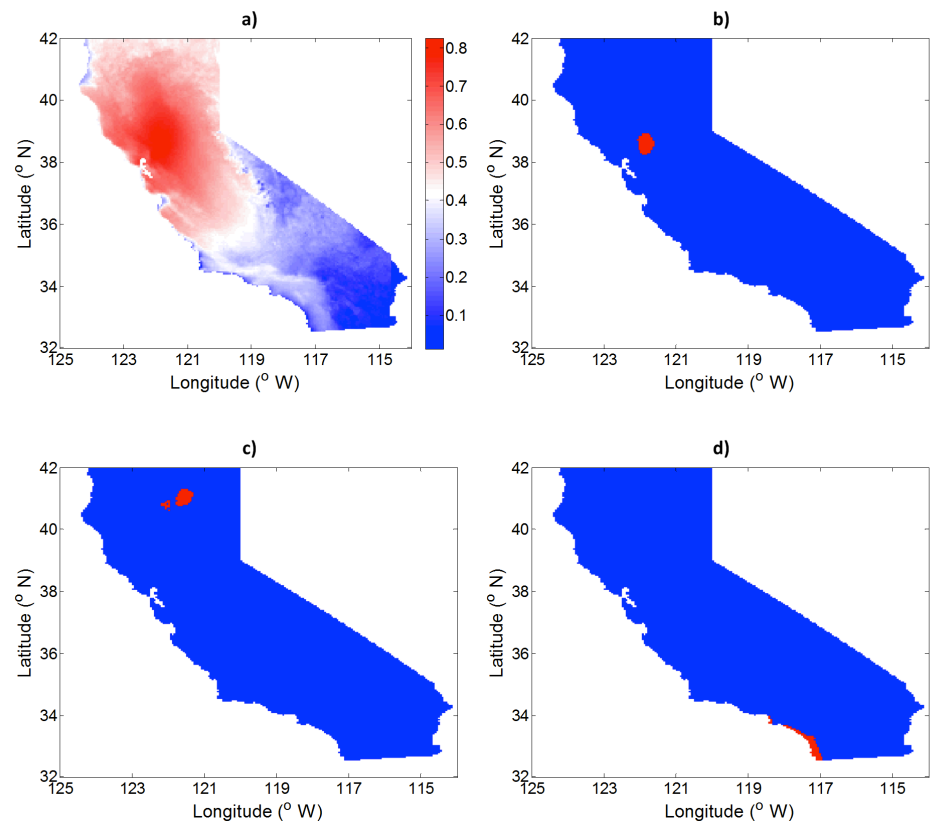

Figure 3: a) Heatmap of the correlation coefficients between the SolarAnywhere ${ }^{\circledR} K$ time series at the California state and the CIMIS station at Davis. The colorbar on the right indicates the magnitude of the Pearson $\rho$ values. The seeking areas for b) Davis, c) McArthur and d) Torrey Pines (San Diego) after applying the threshold of the 100 highest correlated nodes are illustrated in red. The remaining nodes of the CA territory below the threshold values are colored in blue. At these examples no time lag was applied. The correlation analysis is referred to the training subset.

\subsection{Linear models}

The simplest class of forecast modes used in this work are linear models. The general expression for these models is given by 


$$
\begin{aligned}
\hat{k}(t+\Delta t) & =A_{0}+\sum_{i=1}^{N} A_{i} k_{\text {endo }}(t-(i-1) \Delta t) \\
& +\sum_{i=1}^{N} \sum_{j=1}^{M} B_{i j} k_{\text {exo }, i j}(t-(i-1) \Delta t)
\end{aligned}
$$

with $k_{e x o, i j} \in K_{i}$. The coefficients $A_{i}$ and $B_{i j}$ are determined such that they minimize the error

$$
e\left(A_{i}, B_{i j}\right)=\frac{1}{N} \sum_{i=1}^{N}\left|\hat{k}_{i}-k_{i}\right|,
$$

for data points in the training data set. The minimization is done with the quasi-Newton algorithm available through the function fminunc implemented in MATLAB (R3013a). The forecast performance of the various models developed is assessed relatively to the persistence model which is given by Eq. 6 with $A_{1}=1$ and all other coefficients equal to 0 .

\subsection{ANN}

Another class of models tested here are based on ANNs. These are useful tools for approximating complicated mapping functions for problems in classification and regression [25], and have been used extensively in solar radiation modeling and forecasting (see e.g., [26, 27]). An ANN is a particular representation of an output $y$ in terms of some input variables $x$. This representation is based on signals being sent through elements called neurons in such a way that the processing of the input signals produces an output, $\hat{k}$, that is sufficiently close to the desired target value of $k$. Neurons are arranged in layers, where the first layer contains the set of inputs, the last layer contains the output, and the layers in between, referred to as hidden layers, contain hidden neurons. This work uses feedforward neural network with $N_{\text {inp }}$ inputs and $N_{\text {hid }}$ neurons in one hidden layer with a linear output activation. The ANN forecast model can be expressed as,

$$
\hat{k}=\sum_{i=1}^{N_{\text {hid }}} w_{i} f_{i}^{\text {hidden }}\left(\sum_{j=1}^{N_{\text {inp }}} w_{j i} x_{j}+w_{0 i}\right)+w_{0},
$$

where $f_{i}^{\text {hidden }}$ are sigmoidal functions, such as the hyperbolic tangent function. The weights, $w_{j, i}$ and $w_{i}$ in the network were determined with the Levenberg-Marquardt numerical optimization algorithm that minimizes the mean squared error (MSE)

$$
e\left(w_{i}, w_{i j}\right)=\frac{1}{N} \sum_{i=1}^{N}\left(\hat{k}_{i}-k_{i}\right)^{2},
$$

\subsection{Support Vector Regression}

In order to assess the performance of another family of predictors, we use an attractive approach for time series forecasting, the SVR model based on Vapnik's concepts of support vector machines (SVM) [28]. SVR method relies its popularity on characteristics such as the robust and sparse regressions, good generalization performance, unique solution compared to ANN due to the absence of local minima. The basic idea behind SVR is to transform the data $\mathbf{x}$ from an initial input space into a higher dimensional feature space as a means of a nonlinear mapping function $\Phi$ to account for nonlinearity. The transformed feature space maximizes the geometrical margins between the objects allowing the better separation. The hyperplane at the feature space is computed by the linear equation:

$$
f(x)=<w, \Phi(x)>+b,
$$

where $<\cdot, \cdot>$ denotes the inner product, with $w \subset R^{n}, b \subset R$ and $\Phi: R^{n} \rightarrow D$. Initial data $\mathbf{x}$ lay on the $R^{n}$ dimensional space, $D$ is the target feature space, $w$ defines a set of coefficients to be determined and $b$ is the intercept.

The regression problem is equivalent to the optimization problem stated in [28] of finding the parameter vector $w$ and intercept $b$ by requiring the minimization of the equation: 
Table 1: List of possible exogenous variables to include into the forecast models.

\begin{tabular}{llll}
\hline$k_{1}(t)$ & $k_{2}(t)$ & $\cdots$ & $k_{N_{\text {exo }}}(t)$ \\
$k_{1}(t-\Delta t)$ & $k_{2}(t-\Delta t)$ & $\cdots$ & $k_{N_{\text {exo }}}(t-\Delta t)$ \\
$\vdots$ & $\vdots$ & $\ddots$ & $\vdots$ \\
$k_{1}\left(t-\left(N_{\text {lag }}-1\right) \Delta t\right)$ & $k_{2}\left(t-\left(N_{\text {lag }}-1\right) \Delta t\right)$ & $\cdots$ & $k_{N_{\text {exo }}}\left(t-\left(N_{\text {lag }}-1\right) \Delta t\right)$ \\
\hline
\end{tabular}

$$
\min _{\mathbf{w}, b, \xi, \xi^{*}} \frac{1}{2}\|\mathbf{w}\|^{2}+C \sum_{i=1}^{n}\left(\xi_{i}+\xi_{i}^{*}\right),
$$

subject to

$$
\begin{aligned}
& f\left(x_{i}\right)-y_{i} \leq \varepsilon+\xi_{i}, \xi_{i} \geq 0, \forall i, \\
& f\left(x_{i}\right)-y_{i} \geq-\varepsilon-\xi_{i}^{*}, \xi_{i}^{*}, \geq 0, \forall i, \\
& i \geq 1,2, \ldots, N
\end{aligned}
$$

where the regularization parameter $C$ determines the trade-off between the 'flatness' of $f$ and amount up to which deviations larger than the regression margin $\epsilon$ are tolerated. The slack constrains $\xi, \xi^{*}$ are introduced to measure the distance between the input data and outside the $\epsilon$-tube [29]. The solution to the regression problem requires computations of only the above mentioned inner product, which can be presented in the feature space as a kernel function:

$$
f(x)=\sum_{i=1}^{N} \alpha_{i} K\left(x, x_{i}\right)+b,
$$

where $\alpha_{i}$ are Lagrange multipliers associated with the constrains and $K\left(x, x_{i}\right)$ is the introduced kernel function. The Gaussian radial basis function (RBF) is selected among basic kernel choices, defined as:

$$
K\left(x, x_{i}\right)=G\left(\left\|x-x_{i}\right\| ; \gamma\right)=e^{-\gamma\left\|x-x_{i}\right\|},
$$

where $\gamma$ is an user defined parameter that defines the kernel behavior, while $x$ is the center of the Gaussian distribution. Here, the parameters $\gamma$ and $C$ are set equal to 16 and 0.25 , respectively.

\subsection{Selecting exogenous variables}

The models explained in the previous sections receive as inputs the clear-sky index from the ground data and the clear-sky index from the satellite gridded data. After applying the correlation analysis procedure outlined in section 3.1 there are still hundreds of possible inputs to consider at the different time-lags with significant correlation $\rho_{\tau, i} \geq 0.5$. The first approach used to include exogenous variables in the forecast models was an brute force method in which, for each lag correlation map $\rho_{\tau, i}$, all the exogenous variables in the set $\left.K_{\tau}\right|_{\rho_{\tau, l i m}}$ with $\rho_{\tau, \text { lim }}=\left\{\rho_{\tau, 1 \text { st }}, \rho_{\tau, 2 \text { nd }}\right.$, $\left.\cdots, \rho_{\tau, 50 t h}\right\}$. The possible inputs, for those cases, are summarized by Table 1 that list the available inputs for the 4 time lags considered (the table rows) and the $N_{\text {exo }}$ exogenos locations in the set $\left.K_{\tau}\right|_{\rho_{\tau, l i m}}$ (the table columns). In other words, exogenous locations with the highest $1,2, \cdots, 50$ correlations within the seeking area, as it is defined in $\S 3.1$, for each lag were included in to the forecast models, the free parameters computed using the validation dataset and the error metrics computed for the testing set. This approach returned the best forecast models that use all the highest correlated (up to 50) nodes for a single time lag.

In order to involve all the time lags in the same model we used a simple search algorithm:

1. find the best number of highly correlated exogenous data, at lag 0-hours, to add to the forecast algorithm

2. sequentially add highly correlated exogenous data from lag 1-hour to the set found in the previous step and determined the number of exogenous variables (if any) that minimize the forecast error

3. repeat step 2 for the exogenous data at lags 2- and 3-hours. 


\subsection{Genetic algorithms}

The previous section explained a brute force method to include exogenous variables in the forecast. In this section we explain a more general approach to the problem of finding the best set of exogenous inputs based on the heuristic search tool, the genetic algorithm [30]. The GA is especially useful for problems such as this where it is not possible to map the search space analytically. In this work, the GA starts with an initial population of individuals generated randomly, each of which representing a different set of exogenous inputs. Each individual in the population is described by a binary vector with length $N_{L a g} \times N_{E x o}$. The element $i=(j-1) N_{L a g}+k$ in the one of these vectors, where $j$ and $k$ are the row index and column index from Table 1 , respectively, dictates the inclusion (if equal to 1 ) or the exclusion (if equal to 0 ) of the $j k$ input into the forecast model.

All the individuals are then evaluated using the linear forecast explained above. The individuals with the lowest MSE when applied to the optimization dataset are selected as parents for the following generation. Eighty percent of offspring generation is created through crossover (bit-wise random recombination from two parents). The remainder $20 \%$ are created through mutation of randomly selected parents. In this case mutation operates by flipping a bit at some randomly chosen sites. The new generation is again classified in terms of forecast MSE and the process is repeated until there is no more improvement in the best individual or a prescribed number of generations is achieved, 100 in this case.

Due to the stochastic nature of the GA, multiple runs were performed to ensure that the final solutions do not vary significantly depending on the GA run. Figure 4 illustrates the evolution of multiple GA runs for the location of Windsor for the 1 hour forecast horizon. Each of the $10 \mathrm{GA}$ runs start with a random initial population for a fixed number of 100 generations. It is notable in Fig. 4 that the short range of the generated fitness scores is mainly found within the first two standard deviations of the mean, and the upper and lower quartiles are almost included within one standard deviation. Consequently, a single run of the GAs can be used to provide a reliable and fast solution.

a)

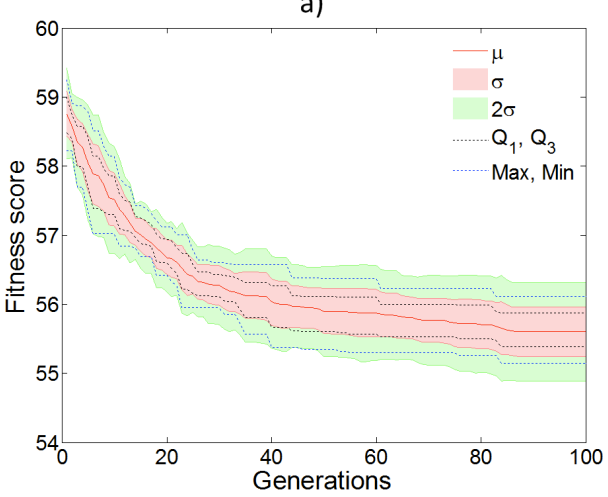

b)

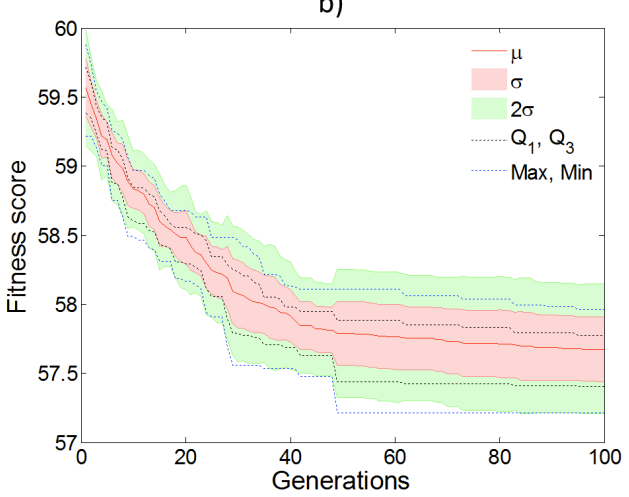

Figure 4: Genetic algorithm performance graphs for $1 \mathrm{~h}$ forecast horizon at Windsor station: a) Summer (Biannual), b) Annual. Statistical measures (mean, standard deviations, upper and lower quartiles, $\max / \mathrm{min}$ data range) of the fitness score (\%) are presented for 10 runs with a random initial population against a learning time of 100 generations.

\section{Forecasting results}

\subsection{Preprocessing and forecast models}

As explained in section $\S 2$, we focus on performing our forecasting models to GHI data selected from ground locations throughout CA referred to as endogenous variables, while satellited-derived GHI data are considered as exogenous variables. A preprocessing step for only daylight timestamps includes the removal of missing values, calculation of the CSM values, normalization to clear sky index $k_{\text {exo/endo }}$ and division of the entire data period into training, validation and testing sets. Subsequently, all of the previously described forecasting methods are applied to predict future values of normalized GHI for $1 \mathrm{~h}, 2 \mathrm{~h}$ and $3 \mathrm{~h}$ ahead forecast horizons. The inputs were either timelagged or synchronous endogenous ground-measured $k$ time-series with/without exogenous variables obtained from the satellite gridded data. 
The experiments in this study were conducted in a three-step procedure. In the first step we conduct a correlation analysis between the selected CIMIS location and the time-lagged satellite-derived $k$ time-series from the training subset to investigate the spatial area of the highest correlated locations. A threshold value of the 100 most wellcorrelated locations around a CIMIS station has previously identified to define the seeking area for each lag.

The second step follows with the application of all the forecasting models to the validation subset for each location of interest. In this step each model goes through a parameter optimization step to ensure the accuracy of the prediction process. All the terms of $1 \mathrm{~h}$-to-3h time-lagged or synchronous (zero time lag) endogenous/exogenous data inputs were examined with respect to the configuration of each model, as described by the Eq. 2. In particular, the linear model was applied either to only endogenous variables or to both endogenous and exogenous input data for the various time lags and forecast horizons. It is worth noting that the seeking area for exogenous variables varies for different locations and at each implementation the models use a different number of exogenous variables within the predefined area of interest, varying gradually from 1 to 50. The upper limit of 50 exogenous variables was selected based on a realistic scenario of choosing a reasonable number of locations that can be potentially used for hosting ancillary ground stations. In brief, we can distinguish five basic variations of the used linear models. First, the simple persistence model, second, the linear model using only endogenous variables and the linear model that uses a combination of endogenous variables accompanied by exogenous variables from a single lag separately. Furthermore, we select to add together the number of exogenous variables that perform best for each lag in order to investigate the effect of applying simultaneously different but also best performing time-lags to the prediction of future values. In this model type, exogenous variables from previous lags are added only if they improve the forecast. Another essential point of using exogenous variables to improve the performance of the linear models, is the type of linear models that uses the GA to determine the optimal set of exogenous variables for each time lag. The heuristic search by the GA applies always within the seeking area derived from the correlation analysis at each time lag and the selected number of variables is independent from a time lag to another. In our case study, experiments with various GA population sizes showed that there is no significant improvement to select more than 50. Finally, the SVR and ANN non-linear forecast models, using only multiple time-lagged endogenous inputs, are used to provide more advanced reference models than the linear ones.

Upon the completion of each model's parameters optimization, the third step of the experimentation is to apply the trained forecasting models to the testing subset. Once the model parameters are determined, we evaluate the models on the testing set in terms of measuring their efficiency to an independent period of input data. Results generated by all the forecast models to both the validation and testing subsets with reference to each CIMIS location. The following subsection provide an overview of the performance metrics.

\subsection{Performance measures}

To evaluate the performance of the developed prediction models, we use the naive persistence model as the reference model. The output of the forecasting models are the predicted $\hat{k}$ time-series for each ground point of interest, which are transformed to predicted GHI values, as defined by the Eq.1. Both for the validation and the testing subsets, the predicted results for each case are compared with the measured observations by calculating the Root Mean Square Error (RMSE) as follows:

$$
R M S E=\sqrt{\sum_{t=1}^{n_{s}} \frac{(\mathcal{P}(t)-\hat{P}(t))^{2}}{n_{s}}}
$$

where $\mathcal{P}$ stands for the measured GHI observations $\left(\mathrm{W} / \mathrm{m}^{2}\right)$ at each ground CIMIS location at time $t, \hat{P}$ denotes the predicted GHI values at the same location and $n_{s}$ is the length of data set.

In order to examine the relative improvement of the different prediction methods over the baseline persistence model, we calculate the model quality skill $s$ defined by:

$$
\operatorname{skill}(\%)=\left(1-\frac{R M S E_{\text {mod }}}{R M S E_{\text {pers }}}\right) \times 100,
$$

where $R M S E_{\text {mod }}$ means the RMSE of each model forecast, while $R M S E_{\text {pers }}$ stands for the RMSE of the persistence forecast model on either the validation or the testing subset. The higher the value of $s$, the better the forecasting model 
against the persistence model performs. Negative values of $s$ indicate that the forecasting model fails to outperform the persistence model, while $s$ equal to 1 denotes a perfect forecast with zero $R M S E_{\text {mod }}$.

In addition, the Coefficient of Determination $\left(R^{2}\right)$ statistical measure is calculated as follows:

$$
R^{2}=1-\frac{\sum_{t=1}^{n_{s}}(\mathcal{P}(t)-\hat{P}(t))^{2}}{\sum_{t=1}^{n_{s}}(\mathcal{P}(t)-\overline{\mathcal{P}}(t))^{2}},
$$

where $\overline{\mathcal{P}}$ is the mean of the measured values $\mathcal{P}$ for the period of each subset. Complementary to the comparative advantage of a forecasting model against a simple reference model as this is calculated by $s$, the Coefficient of Determination scores between 0 and 1 indicate the extent to which the predicted $\hat{P}$ variables are predictable from the measured values $\mathcal{P}$. In other words, the $R^{2}$ values show how accurately the predicted points fall on a simple regression line (See Fig 7). This metric evaluates the performance of each model independently of the reference model's performance and provides insight into the reliability of the model itself.

\subsection{Seasonal-dependent optimization}

In the initial experiments the models were developed using the entire dataset as explained above. This results that the free parameters in the different models are optimized for data belonging to the whole year and all types of weather conditions. It is then possible, that if the models are retrained for smaller sets of more coherent data the performance may increase. To study this effect the original data sets are divided with respect to seasons in a very simple way:

- quarterly, the sets are divided into four meteorological seasons defined as i) Winter (Dec-Jan-Feb), ii) Spring (Mar-Apr-May), iii) Summer (Jun-Jul-Aug) and iv) Autumn (Sep-Oct-Nov).

- biannual, the sets are divide into two major subsets i) Nov-Mar and ii) Apr-Oct. This selection is contingent on empirical regional conditions.

- annual, data from the entire year forms either the validation or the testing set.

\subsection{Forecasting performance}

Tables 3-11 present the performance results in terms of forecast skill $s$ and $R M S E$ with respect to the three seasonal-dependent types for partitioning the evaluation period of time (i.e. validation and testing subsets) as well as the forecast horizon. As the Tables show, each row indicates a different model, while each column corresponds to a different CIMIS ground station. Metric values are given for both the validation and the testing subsets. Whereas the experiments are conducted on a seasonal basis taking into consideration the aforementioned division scenarios, the performance metrics are calculated accordingly. In order to simplify the presentation of the results, we thereafter merge the forecasts into an annual basis while ensuring that the merged timestamps are distinct. Consequently, the following figures and tables display the merged results obtained by different forecast methods and partition of the evaluation subsets.

For better visualization of the results at Tables 3-11, we plot the content of each table in a radial plot in Fig. 5. The measured $s$-skill scores for the testing subset for each forecasting model can be read by tracing an axis from the center outwards, each axis corresponds to one of the 7 CIMIS ground locations. Therefore, values obtained from the same method across different ground stations can be connected by a joined line and the corresponding area is shaded by different colors to increase the distinction. We label the axes in the prescribed geographical orientation of the ground stations, starting clockwise with McArthur (northern) to Torrey Pines (southern).

Figure 6 shows the bar plots of $s$-skill values for the best performing forecasting method, the linear model optimized by the GA, both at each quarter of the four-seasonal division of the evaluation subsets and also the merged scheme. In these plots, each set of bars corresponds either to the seasonal or the merged results, while the color of each bar stands for different prediction horizon and evaluation subset. From this figure it can be seen that the forecasting performance varies from a season to another, depending also on different factors such as the location of the CIMIS stations and the evaluation subset in use.

By plotting the pairs of forecasted $\hat{P}$ values and measured $\mathcal{P}$ observations of the testing subset for specific ground stations, we produced the scatter plots at Fig. 7. As can be seen from the scatter plots, the Coefficient of Determination 
$\left(R^{2}\right)$ values are calculated to quantify the degree of dispersion of the forecasted values about the 1:1 reference line. The highest values of $R^{2}$, the better performance of the forecasting method. The upper half set of scatter plots outlines the performance of the ground station with the best results (i.e. De Laveaga) and the bottom half plots refer to the less well performing station (i.e. Parlier). As the key aspect of using the $R^{2}$ metric is to measure the accuracy of a forecasting model autonomously rather than comparatively to a reference model, the scatter plots correspond to the best performing and the reference model, as these are derived from our experiments. Hence, the left column of the 7 corresponds to the linear model optimized by GA and the right column corresponds to the persistence reference model. The rows show examples for 1 and 3 hours ahead forecastings. We also indicate the quality of the forecast in terms of RMSE above each scatter plot. From this figure it can be seen that the depicted performance of each case is relevant to the corresponding $s$-skill metric; the linear model optimized by the GA performs always better than the persistence model. It is obvious that both the location of the point of interest and the forecasting horizon affect the forecasting quality.

\section{Conclusions}

This study shows that the forecasting accuracy for GHI in the state of California can be substantially increased through exogenous, satellite-derived data (such as the data provided by the gridded SolarAnywhere ${ }^{\circledR}$ dataset). Given the costs associated with ground-based station networks, the proposed methodology leverages the availability of lowcost satellite data in such way that the forecasts can be improved at any point of interest. To this end, we: i) propose a time-lagged correlation analysis to define precisely the seeking area of the exogenous satellite inputs, and ii) demonstrate that the linear model optimized by GA outperforms any other method tested here. As for the forecasting improvements by means of $s$-skill, the GA optimized model outperforms the persistent model by 8.458 to $40.419 \%$ on the quarterly basis, 8.185 to $41.180 \%$ on the biannual basis and 8.969 to $39.196 \%$ on the annual basis, depending on the forecast horizon and station location. Summing up the results, we conclude that a combination of hourly-shifted inputs (up to $3 \mathrm{~h}$ ) before the current time $t$ is critical for forecast improvements. We also determine the optimum number of exogenous variables per lag.

The seasonal partition of the evaluation subsets shows that there are periodic tendencies of the irradiance input for all the models except the best performing model, which performs fairly unbiased for all seasons. The results shown in the Fig. 5 indicate that the shape of the patterns are significantly altered in season-dependent divisions, in contrast to that of the best model, which remains robust for certain forecast horizons.

An optimal spatial distribution for sensor networks can be determined through direct application of the top performing forecasting model. Points of interest located near the coastline (e.g. De Laveaga, Windsor, Torrey Pines) exhibit better accuracy compared to the forecast improvements of mostly dry, cloudless inland locations such as Merced, Davis, Parlier. With respect to the quarterly seasonal results (Fig. 6), the coastal locations perform considerably better during summer and autumn as compared to the most dynamic periods in winter and spring. The the negative $s$-skill result for the summer quarter at Davis can be explained as input data discrepancies from different sources. Our findings also reveal the weakness of the reference model to perform well as the forecast horizon progresses. It is apparent that the well performing locations exhibit a more distinct forecast improvement against the reference model at larger forecast horizons (see Fig. 7).

The proposed methodology can be readily adapted to existing networks of ground stations or to a single ground station that is covered by high-resolution satellite imagery. Although several studies have indicated the use of exogenous variables derived by neighboring stations of an existing station, the innovation of this study is in the exploitation of satellite-derived inputs within a gridded domain to generate accuracy and robustness in the forecasts. Hence, the ultimate proximity that can be reached between the point of interest and the potential exogenous variables, together with the localization of the seeking area and the optimization of time-lagged exogenous variables, suggests a reliable approach towards the development of more accurate and robust solar forecasts.

\section{Acknowledgments}

The authors gratefully acknowledge the partial financial support given by the DOE SUNRISE award DE-EE0006330, by the California Public Utilities Commission (CPUC) under the California Solar Initiative (CSI) Program, and the 
partial support by the National Science Foundation (NSF) EECS (EPAS) award N. 1201986, which is managed by Dr. Paul Werbos.

\section{References}

[1] R. H. Inman, H. T. C. Pedro, C. F. M. Coimbra, Solar forecasting methods for renewable energy integration, Progress in Energy and Combustion Science 39 (6) (2013) 535-576.

[2] R. Marquez, V. G. Gueorguiev, C. F. M. Coimbra, Forecasting of global horizontal irradiance using sky cover indices, Journal of Solar Energy Engineering 135 (1) (2012) 011017-011017.

[3] Y. Chu, H. T. C. Pedro, C. F. M. Coimbra, Hybrid intra-hour DNI forecasts with sky image processing enhanced by stochastic learning, Solar Energy 98, Part C (2013) 592-603.

[4] R. Marquez, H. T. C. Pedro, C. F. M. Coimbra, Hybrid solar forecasting method uses satellite imaging and ground telemetry as inputs to ANNs, Solar Energy 92 (2013) 176-188.

[5] V. P. A. Lonij, A. E. Brooks, A. D. Cronin, M. Leuthold, K. Koch, Intra-hour forecasts of solar power production using measurements from a network of irradiance sensors, Solar Energy 97 (2013) 58-66.

[6] D. Yang, C. Gu, Z. Dong, P. Jirutitijaroen, N. Chen, W. M. Walsh, Solar irradiance forecasting using spatial-temporal covariance structures and time-forward kriging, Renewable Energy 60 (2013) $235-245$.

[7] A. Sözen, E. Arcaklığlu, M. Özalp, N. Çağlar, Forecasting based on neural network approach of solar potential in Turkey, Renewable Energy 30 (7) (2005) $1075-1090$.

[8] S. Pelland, G. Galanis, G. Kallos, Solar and photovoltaic forecasting through post-processing of the Global Environmental Multiscale numerical weather prediction model, Progress in Photovoltaics: Research and Applications 21 (3) (2013) 284-296.

[9] S. Al-Alawi, H. Al-Hinai, An ANN-based approach for predicting global radiation in locations with no direct measurement instrumentation, Renewable Energy 14 (14) (1998) 199 - 204.

[10] A. Nottrott, J. Kleissl, Validation of the NSRDB-SUNY global horizontal irradiance in california, Solar Energy 84 (10) (2010) $1816-1827$.

[11] L. Nonnenmacher, A. Kaur, C. F. M. Coimbra, Verification of the SUNY direct normal irradiance model with ground measurements, Solar Energy 99 (2014) 246-258.

[12] Geostationary Operational Environmental Satellite R-Series, User Systems: GOES-R Rebroadcast (2014). URL http: //www.goes-r.gov/users/grb.html

[13] Solar Anywhere, SolarAnywhere data, Clean Power Research 2012 (2012) URL http: //www. solaranywhere.com

[14] R. Perez, P. Ineichen, K. Moore, M. Kmiecik, C. Chain, R. George, F. Vignola, A new operational model for satellite-derived irradiances: description and validation, Solar Energy 73 (5) (2002) 307-317.

[15] R. Perez, P. Ineichen, M. Kmiecik, K. Moore, D. Renne, R. George, Producing satellite-derived irradiances in complex arid terrain, Solar Energy 77 (4) (2004) $367-371$

[16] F. Vignola, P. Harlan, R. Perez, M. Kmiecik, Analysis of satellite derived beam and global solar radiation data, Solar Energy 81 (6) (2007) $768-772$.

[17] P. Ineichen, Comparison of eight clear sky broadband models against 16 independent data banks, Solar Energy 80 (4) (2006) $468-478$.

[18] R. H. Inman, H. T. C. Pedro, C. F. M. Coimbra, Solar forecasting methods for renewable energy integration, Prog. Energy Combust. Sci. 39 (2013) $535-576$.

[19] P. Ineichen, R. Perez, A new airmass independent formulation for the linke turbidity coefficient, Solar Energy 73 (3) (2002) $151-157$.

[20] A. Zagouras, R. H. Inman, C. F. M. Coimbra, On the determination of coherent solar microclimates for utility planning and operations, Solar Energy 102 (2014) 173-188.

[21] V. Badescu, C. A. Gueymard, S. Cheval, C. Oprea, M. Baciu, A. Dumitrescu, F. Iacobescu, I. Milos, C. Rada, Accuracy analysis for fifty-four clear-sky solar radiation models using routine hourly global irradiance measurements in romania, Renewable Energy 55 (2013) 85 - 103.

[22] C. N. Long, T. P. Ackerman, Surface measurements of solar irradiance: a study of the spatial correlation between simultaneous measurements at separated sites, J. Appl. Meteor. 34 (1995) 1039-1046.

[23] C. Glasbey, R. Graham, A. Hunter, Spatio-temporal variability of solar energy across a region: a statistical modelling approach, Solar Energy 70 (4) (2001) $373-381$.

[24] A. Mellit, Artificial intelligence technique for modelling and forecasting of solar radiation data: a review, Int. J. Artif. Intell. Soft Comput. 1 (1) (2008) 52-76

[25] C. Bishop, Neural networks and their applications, Review of Scientific Instruments 65 (1994) 1803-1832.

[26] H. T. C. Pedro, C. F. M. Coimbra, Assessment of forecasting techniques for solar power production with no exogenous inputs, Solar energy 86 (7) (2012) 2017-2028.

[27] A. Mellit, M. Benghanem, S. Kalogirou, Modeling and simulation of a stand-alone photovoltaic system using an adaptive artificial neural network: Proposition for a new sizing procedure, Renewable Energy 32 (2) (2007) 285-313.

[28] V. N. Vapnik, The Nature of Statistical Learning Theory, Springer-Verlag New York, Inc., New York, NY, USA, 1995.

[29] B. Scholkopf, A. J. Smola, Learning with Kernels: Support Vector Machines, Regularization, Optimization, and Beyond, MIT Press, Cambridge, MA, USA, 2001.

[30] J. H. Holland, Adaptation in natural and artificial systems, Ann Arbor MI, University of Michigan Press, 1975. 
a)

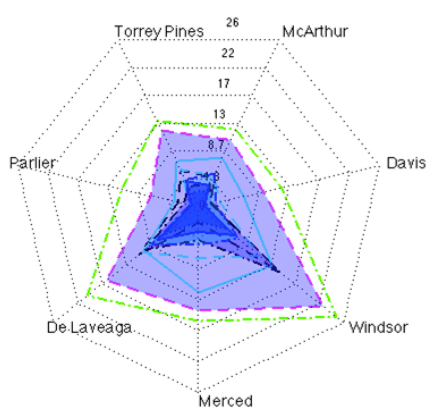

d)

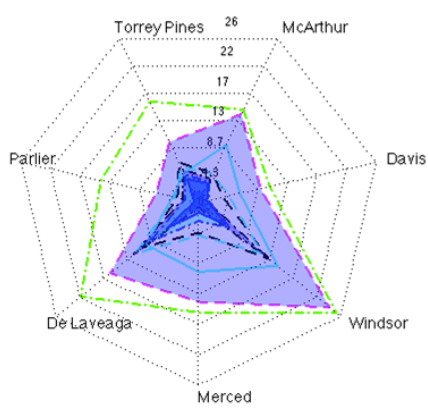

g)

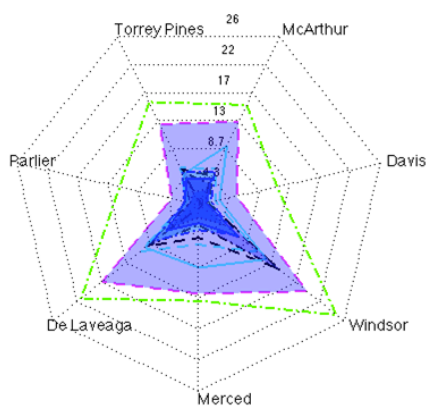

b)

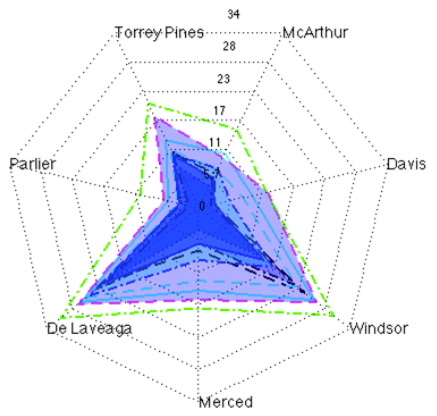

e)

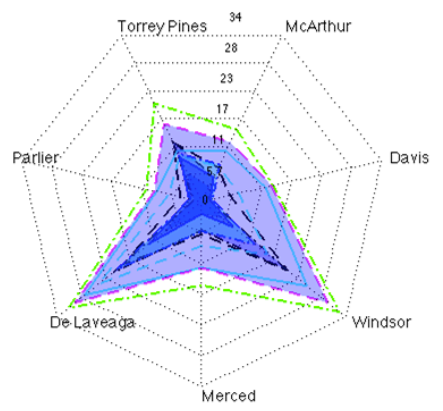

h)

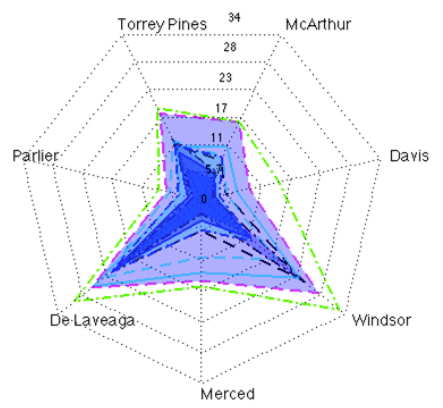

c)

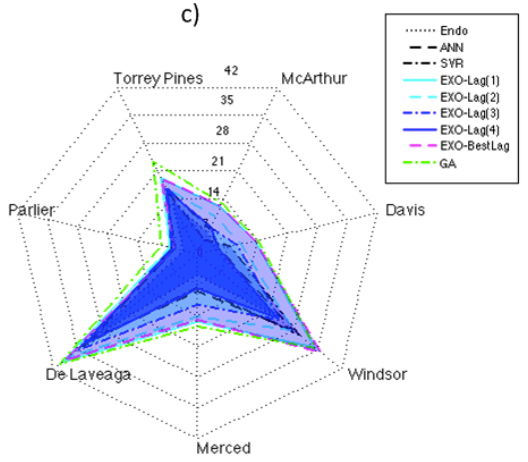

f)
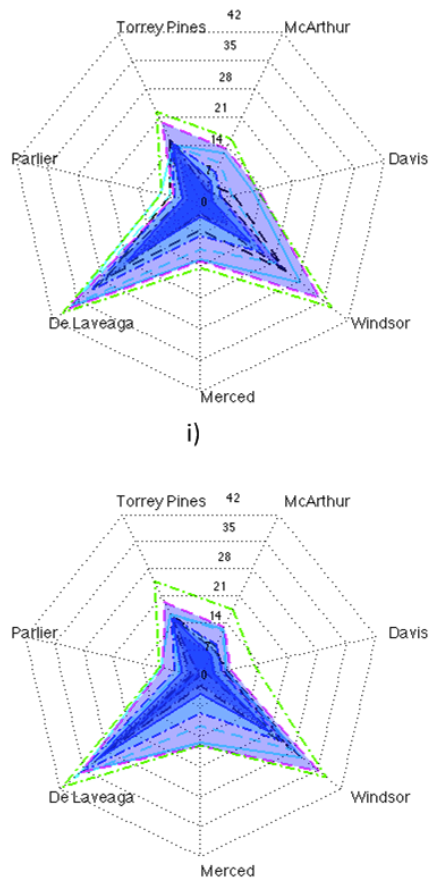

Figure 5: Radial plot of the performance metric $s(\%)$. The merged scores of the $s$-skill are shown for each station location (radial axes) with clockwise north-to-south orientation starting from the north (McArthur). The legend indicates the 9 forecasting models applied to the testing subset. Plots are displayed according to the subset division (quarterly, annual, biannual) and the forecasting horizon in hours (h) as follows: a) Quarterly - 1h, b) Quarterly - 2h, c) Quarterly - 3h, d) Annual - 1h, e) Annual - 2h, f) Annual - 3h, g) Biannual - 1h, h) Biannual - 2h, i) Biannual-3h. 

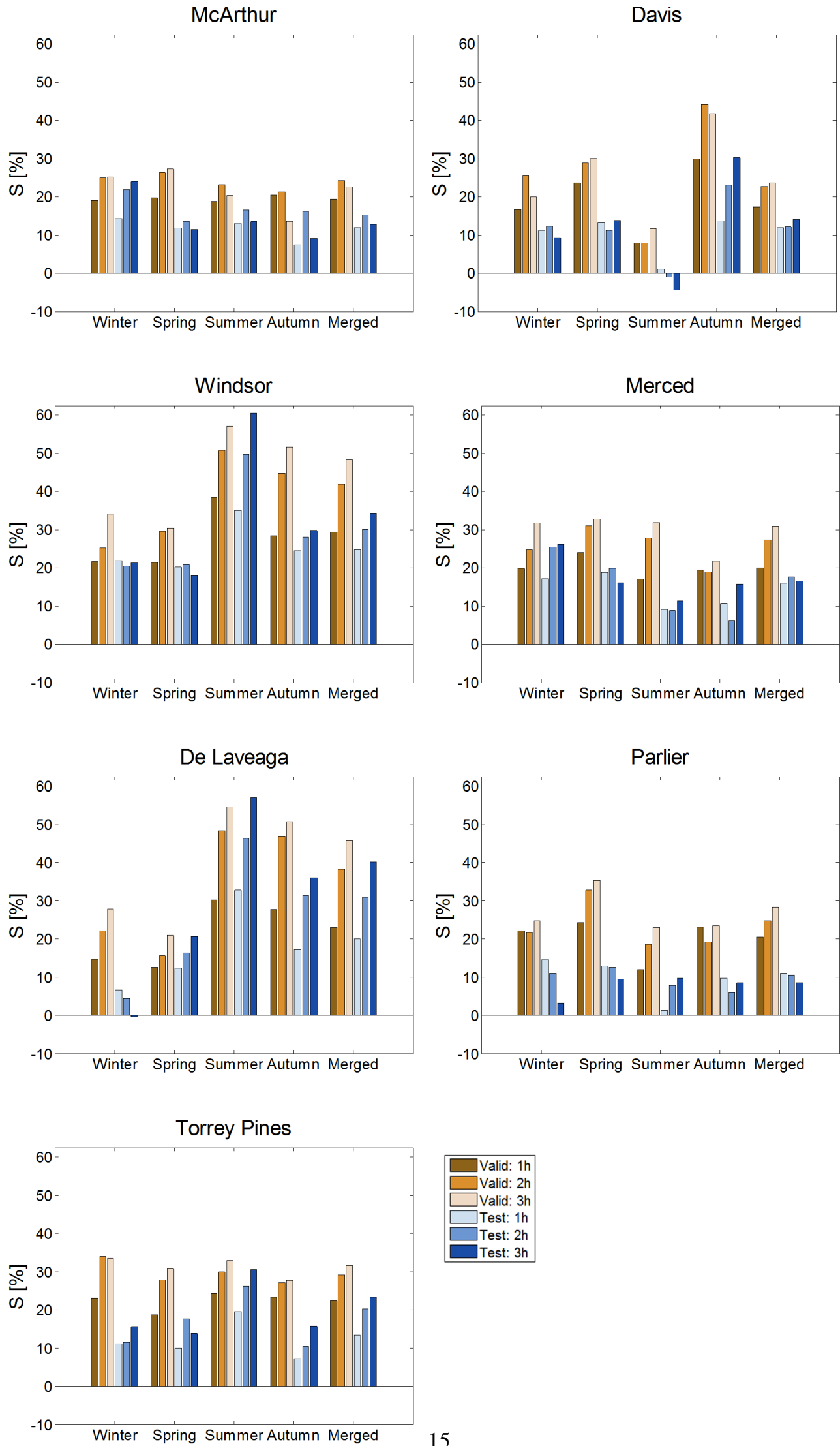
a)

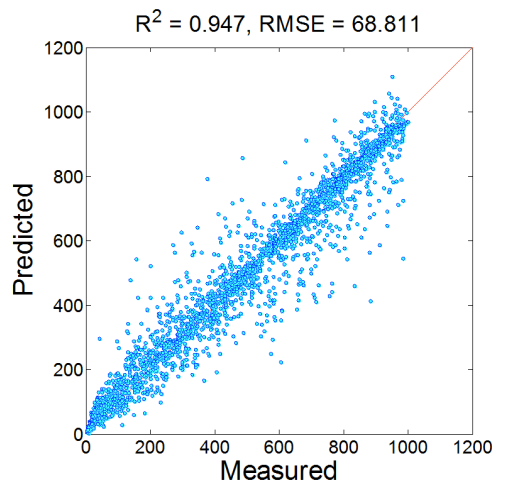

c)

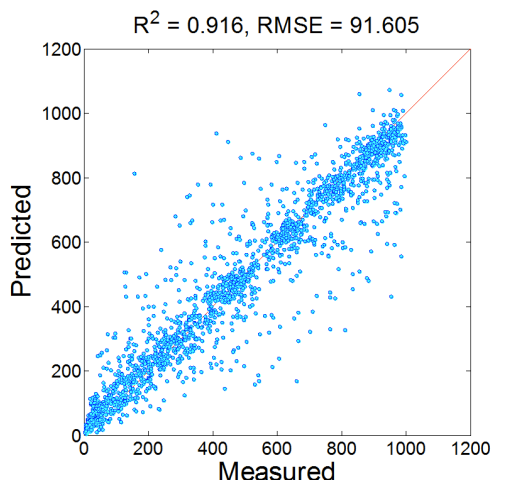

e)

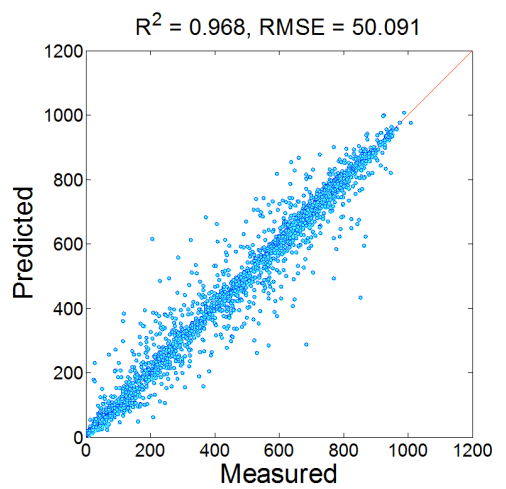

g)

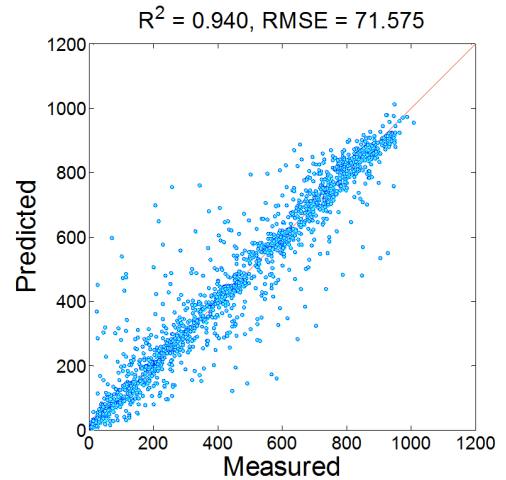

b)

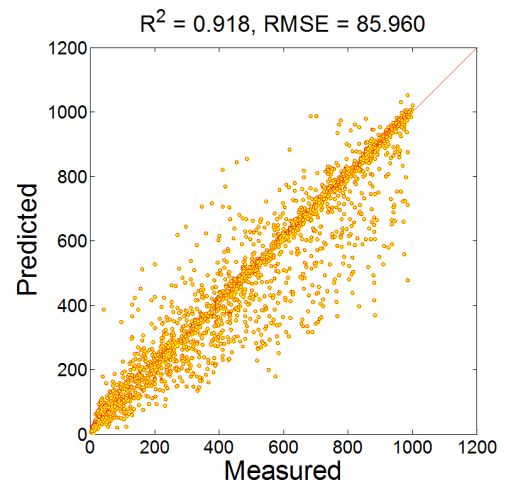

d)

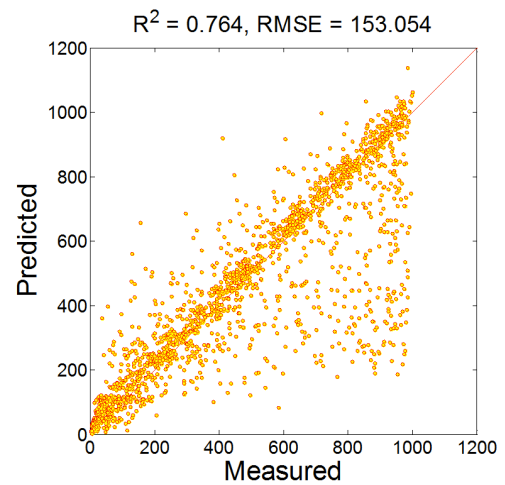

f)

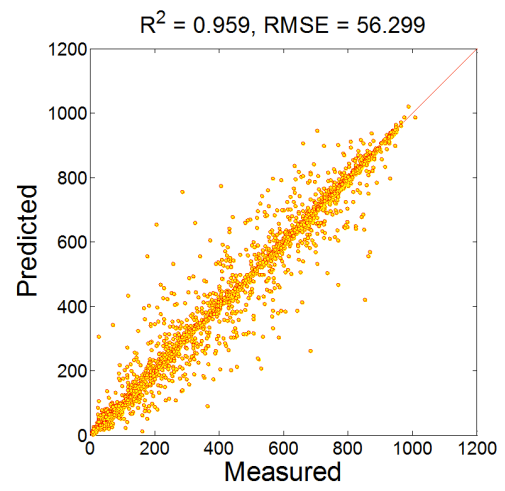

h)

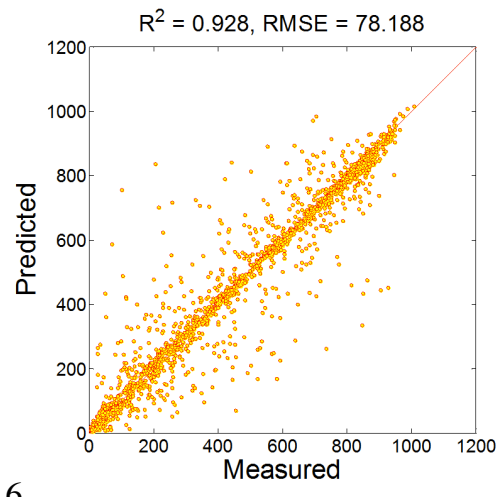

Figure 7: Scatter plots of measured and predicted measurements of the testing subset as they divided into a quarterly seasonal basis. The predicted measurements are relating to the merged results for the following Location - Forecast Horizon - Model: a) De Laveaga - 1h - GA, b) De Laveaga 1h - Persistence, c) De Laveaga - 3h - GA, d) De Laveaga - 3h - Persistence, e) Parlier - 1h - GA, f) Parlier - 1h - Persistence, g) Parlier - 3h - GA, h) Parlier - 3h - Persistence. The $s$-skill (\%) values for each row are 19.950, 40.149, 11.027, 8.458, respectively. The coefficient of determination (R-square) and the RMSE are also shown above each plot. Solid red lines are referred to the 1:1 reference lines. 
Table .2: Characteristics of CIMIS Weather Stations (sorted by North to South).

\begin{tabular}{c|ccccccc}
\hline No. & CIMIS ID & $\begin{array}{c}\text { Station } \\
\text { name }\end{array}$ & Latitude $\left(^{\circ}\right)$ & Longitude $\left(^{\circ}\right)$ & Elevation $(\mathrm{ft})$ & $\begin{array}{c}\text { Irrigation } \\
\text { region }\end{array}$ & County \\
\hline 1 & 43 & McArthur & $41^{\circ} 3^{\prime} 53^{\prime \prime}$ & $-121^{\circ} 27^{\prime} 16^{\prime \prime}$ & 3310 & Northeast Plateau & Shasta \\
2 & 6 & Davis & $38^{\circ} 32^{\prime} 9^{\prime \prime}$ & $-121^{\circ} 46^{\prime} 32^{\prime \prime}$ & 60 & Sacramento Valley & Yolo \\
3 & 103 & Windsor & $38^{\circ} 31^{\prime} 35^{\prime \prime}$ & $-122^{\circ} 49^{\prime} 42^{\prime \prime}$ & 85 & North Coast Valleys & Sonoma \\
4 & 148 & Merced & $37^{\circ} 18^{\prime} 49^{\prime \prime}$ & $-120^{\circ} 23^{\prime} 11^{\prime \prime}$ & 200 & San Joaquin Valley & Merced \\
5 & 104 & De Laveaga & $36^{\circ} 59^{\prime} 52^{\prime \prime}$ & $-121^{\circ} 59^{\prime} 45^{\prime \prime}$ & 300 & Monterey Bay & Santa Cruz \\
6 & 39 & Parlier & $36^{\circ} 35^{\prime} 51^{\prime \prime}$ & $-119^{\circ} 30^{\prime} 15^{\prime \prime}$ & 337 & San Joaquin Valley & Fresno \\
7 & 173 & Torrey Pines & $32^{\circ} 54^{\prime} 4^{\prime \prime}$ & $-117^{\circ} 15^{\prime} 0^{\prime \prime}$ & 335 & South Coast Valleys San Diego \\
\hline
\end{tabular}


Table .3: Summary of the forecast skill and the RMSE statistical error merged results for 1h-ahead horizon on a quarterly basis. The acronyms PERS, ENDO, ANN, SVR, EXO_1 to EXO_4, EXO_best and GA stand for the persistence, the linear model using only endogenous variables (CIMIS locations), the ANN, the SVR, the linear models using exogenous variables from a single lag ( $0 \mathrm{~h}, 1 \mathrm{~h}, 2 \mathrm{~h}$ and $3 \mathrm{~h}$ time shifts, respectively), the linear model that uses the best exogenous variables from each of the time lags, and the linear model optimized by the GA, respectively. The column labels 'Val' and 'Test' indicate the validation and testing subsets.

\begin{tabular}{|c|c|c|c|c|c|c|c|c|c|c|c|c|c|c|}
\hline \multirow[t]{2}{*}{ s $(\%)$} & \multicolumn{2}{|c|}{ Torrey Pines } & \multicolumn{2}{|c|}{ De Laveaga } & \multicolumn{2}{|c|}{ Windsor } & \multicolumn{2}{|c|}{ McArthur } & \multicolumn{2}{|c|}{ Davis } & \multicolumn{2}{|c|}{ Merced } & \multicolumn{2}{|c|}{ Parlier } \\
\hline & Val & Test & Val & Test & Val & Test & Val & Test & Val & Test & Val & Test & Val & Test \\
\hline ENDO & 1.922 & 2.288 & 6.394 & 6.435 & 5.686 & 5.051 & 2.074 & 2.484 & 0.671 & 1.145 & 2.498 & 2.078 & 1.840 & 1.119 \\
\hline ANN & 7.183 & 5.656 & 9.267 & 10.768 & 11.117 & 14.502 & 1.876 & 4.644 & -3.734 & 1.996 & 2.254 & 2.167 & 1.318 & 2.915 \\
\hline SVR & 6.346 & 3.162 & 8.963 & 9.539 & 10.134 & 14.704 & 2.129 & 3.700 & 0.537 & 1.777 & -3.650 & 4.636 & 1.809 & 2.130 \\
\hline EXO_1 & 6.835 & 7.106 & 9.036 & 9.763 & 16.026 & 13.214 & 8.878 & 7.650 & 8.088 & 6.502 & 11.712 & 11.983 & 8.671 & 3.491 \\
\hline EXO_2 & 5.691 & 5.786 & 9.374 & 10.180 & 14.405 & 10.951 & 7.123 & 4.889 & 4.629 & 3.075 & 7.136 & 7.122 & 7.135 & 1.188 \\
\hline EXO_3 & 5.274 & 4.208 & 9.168 & 8.334 & 10.891 & 7.124 & 4.758 & 5.194 & 2.932 & 2.428 & 4.596 & 5.285 & 4.361 & 1.271 \\
\hline EXO_4 & 5.130 & 4.018 & 8.692 & 7.455 & 7.847 & 6.343 & 3.382 & 3.375 & 1.692 & 0.896 & 3.408 & 3.475 & 2.504 & 1.008 \\
\hline EXO_best & 15.172 & 11.893 & 16.009 & 16.535 & 23.054 & 22.345 & 11.997 & 10.398 & 10.616 & 9.383 & 14.445 & 14.518 & 12.461 & 6.857 \\
\hline GA & 22.318 & 13.413 & 23.070 & 19.950 & 29.258 & 24.699 & 19.398 & 12.041 & 17.309 & 11.953 & 20.004 & 15.984 & 20.480 & 11.027 \\
\hline
\end{tabular}

\begin{tabular}{|c|c|c|c|c|c|c|c|c|c|c|c|c|c|}
\hline $\begin{array}{c}\text { RMSE } \\
\left(\mathbf{W} / m^{2}\right)\end{array}$ & & & & & & & & & & & & & \\
\hline PERS 82.695 & 82.680 & 80.997 & 85.960 & 77.045 & 72.112 & 81.603 & 78.115 & 66.283 & 57.200 & 68.124 & 72.160 & 58.727 & 56.299 \\
\hline ENDO 81.105 & 80.788 & 75.818 & 80.429 & 72.664 & 68.469 & 79.911 & 76.175 & 65.838 & 56.546 & 66.422 & 70.661 & 57.647 & 55.668 \\
\hline ANN 76.755 & 78.003 & 73.491 & 76.704 & 68.480 & 61.654 & 80.072 & 74.488 & 68.758 & 56.059 & 66.589 & 70.596 & 57.953 & 54.657 \\
\hline SVR 77.447 & 80.065 & 73.737 & 77.761 & 69.238 & 61.508 & 79.865 & 75.226 & 65.927 & 56.184 & 70.611 & 68.815 & 57.665 & 55.100 \\
\hline EXO_1 77.042 & 76.804 & 73.678 & 77.568 & 64.698 & 62.583 & 74.358 & 72.140 & 60.922 & 53.481 & 60.146 & 63.513 & 53.635 & 54.333 \\
\hline EXO_2 77.989 & 77.896 & 73.404 & 77.209 & 65.947 & 64.214 & 75.790 & 74.296 & 63.215 & 55.442 & 63.263 & 67.021 & 54.537 & 55.630 \\
\hline EXO_3 78.334 & 79.200 & 73.571 & 78.796 & 68.654 & 66.974 & 77.720 & 74.058 & 64.339 & 55.811 & 64.993 & 68.347 & 56.167 & 55.583 \\
\hline EXO_4 78.452 & 79.357 & 73.957 & 79.552 & 70.999 & 67.537 & 78.843 & 75.479 & 65.161 & 56.688 & 65.803 & 69.652 & 57.257 & 55.731 \\
\hline EXO_best 70.149 & 72.846 & 68.030 & 71.747 & 59.283 & 55.998 & 71.813 & 69.993 & 59.246 & 51.833 & 58.284 & 61.684 & 51.409 & 52.438 \\
\hline GA 64.239 & 71.590 & 62.311 & 68.811 & 54.503 & 54.301 & 65.774 & 68.710 & 54.810 & 50.363 & 54.496 & 60.626 & 46.700 & 50.091 \\
\hline
\end{tabular}

Table .4: Same as in Table .3, but for $2 \mathrm{~h}$-ahead horizon on a quarterly basis.

\begin{tabular}{|c|c|c|c|c|c|c|c|c|c|c|c|c|c|c|}
\hline \multirow[t]{2}{*}{ s $(\%)$} & \multicolumn{2}{|c|}{ Torrey Pines } & \multicolumn{2}{|c|}{ De Laveaga } & \multicolumn{2}{|c|}{ Windsor } & \multicolumn{2}{|c|}{ McArthur } & \multicolumn{2}{|c|}{ Davis } & \multicolumn{2}{|c|}{ Merced } & \multicolumn{2}{|c|}{ Parlier } \\
\hline & Val & Test & Val & Test & Val & Test & Val & Test & Val & Test & Val & Test & Val & Test \\
\hline ENDO & 6.820 & 7.873 & 16.070 & 20.443 & 12.615 & 13.131 & 4.093 & 4.286 & 1.785 & 2.950 & 4.449 & 3.918 & 4.700 & 2.337 \\
\hline $\mathbf{A N N}$ & 10.936 & 10.774 & 16.776 & 20.512 & 17.157 & 23.895 & 5.515 & 6.634 & -2.981 & 2.879 & -0.547 & 7.465 & 5.166 & 1.703 \\
\hline SVR & 11.539 & 11.135 & 17.206 & 22.835 & 14.739 & 21.678 & 3.693 & 5.556 & 3.444 & 2.157 & 1.385 & 6.232 & 3.587 & 3.104 \\
\hline EXO_1 & 13.347 & 13.178 & 28.014 & 26.390 & 31.394 & 25.868 & 13.365 & 10.602 & 12.078 & 9.640 & 16.137 & 14.492 & 14.911 & 5.445 \\
\hline EXO_2 & 12.348 & 11.253 & 25.627 & 25.122 & 24.928 & 21.971 & 9.784 & 7.423 & 9.349 & 8.003 & 13.738 & 12.764 & 13.057 & 3.689 \\
\hline EXO_3 & 11.941 & 10.284 & 21.015 & 24.752 & 17.498 & 16.573 & 8.331 & 7.698 & 5.227 & 5.623 & 9.205 & 9.127 & 9.342 & 3.261 \\
\hline EXO_4 & 10.421 & 10.148 & 17.531 & 21.004 & 14.580 & 14.641 & 6.153 & 5.862 & 4.389 & 2.812 & 6.169 & 6.284 & 6.482 & 3.495 \\
\hline EXO_best & 19.636 & 17.852 & 30.683 & 27.028 & 33.530 & 26.460 & 15.213 & 9.817 & 15.922 & 12.443 & 19.722 & 16.114 & 17.192 & 6.255 \\
\hline GA & 29.185 & 20.312 & 38.299 & 30.884 & 41.913 & 30.169 & 24.284 & 15.284 & 22.740 & 12.220 & 27.261 & 17.593 & 24.741 & 10.495 \\
\hline \multicolumn{15}{|l|}{$\begin{array}{c}\text { RMSE } \\
\left(\mathbf{W} / m^{2}\right)\end{array}$} \\
\hline PERS & & 120.273 & & & 111.829 & 101.584 & 105.982 & & 85.274 & & 87.340 & 85.355 & 75.313 & 72.459 \\
\hline ENDO & 108.447 & 110.804 & 101.950 & 101.853 & 97.722 & 88.244 & 101.644 & 94.348 & 83.752 & 68.727 & 83.454 & 82.010 & 71.773 & 70.766 \\
\hline $\mathbf{A N N}$ & 103.657 & 107.315 & 101.092 & 101.765 & 92.643 & 77.311 & 100.138 & 92.034 & 87.815 & 68.777 & 87.818 & 78.983 & 71.423 & 71.225 \\
\hline SVR & 102.955 & 106.880 & 100.570 & 98.790 & 95.346 & 79.563 & 102.068 & 93.096 & 82.337 & 69.289 & 86.130 & 80.035 & 72.612 & 70.210 \\
\hline EXO_1 & 100.851 & 104.423 & 87.442 & 94.240 & 76.722 & 75.306 & 91.817 & 88.123 & 74.974 & 63.989 & 73.246 & 72.985 & 64.083 & 68.514 \\
\hline EXO_2 & 102.014 & 106.738 & 90.341 & 95.862 & 83.953 & 79.264 & 95.613 & 91.256 & 77.301 & 65.149 & 75.341 & 74.460 & 65.479 & 69.786 \\
\hline EXO_3 & 102.487 & 107.903 & 95.944 & 96.336 & 92.262 & 84.749 & 97.153 & 90.985 & 80.816 & 66.834 & 79.301 & 77.565 & 68.277 & 70.097 \\
\hline EXO_4 & 104.256 & 108.067 & 100.175 & 101.135 & 95.524 & 86.711 & 99.461 & 92.794 & 81.531 & 68.825 & 81.952 & 79.991 & 70.432 & 69.927 \\
\hline EXO_best & 93.532 & 98.802 & 84.200 & 93.422 & 74.333 & 74.705 & 89.859 & 88.896 & 71.696 & 62.004 & 70.115 & 71.601 & 62.366 & 67.927 \\
\hline GA & 82.418 & 95.843 & 74.949 & 88.486 & 64.958 & 70.937 & 80.246 & 83.507 & 65.882 & 62.162 & 63.530 & 70.339 & 56.680 & 64.855 \\
\hline
\end{tabular}


Table .5: Same as in Table .3, but for 3h-ahead horizon on a quarterly basis.

\begin{tabular}{rcccccccccrrrrr}
\hline s $(\%)$ & \multicolumn{2}{c}{ Torrey Pines } & \multicolumn{2}{c}{ De Laveaga } & \multicolumn{2}{c}{ Windsor } & \multicolumn{2}{c}{ McArthur } & \multicolumn{2}{c}{ Davis } & \multicolumn{2}{c}{ Merced } \\
\hline & Val & Test & \multicolumn{1}{c}{ Val } & Test & Val & Test & Val & Test & Val & Test & Val & Test & Val & Test \\
\hline ENDO & 10.992 & 13.264 & 20.011 & 31.019 & 10.550 & 22.793 & 5.768 & 5.311 & 3.086 & 4.510 & 6.451 & 4.769 & 6.187 & 3.305 \\
ANN & 14.850 & 17.172 & 23.853 & 31.068 & 24.424 & 27.787 & 6.207 & 6.284 & 1.933 & 5.173 & 6.349 & 8.498 & 5.361 & 5.242 \\
SVR & 15.039 & 15.903 & 26.657 & 33.762 & 24.162 & 29.671 & 4.473 & 3.580 & 4.679 & 7.690 & 3.236 & 7.581 & 3.949 & 5.687 \\
EXO_1 & 16.583 & 19.171 & 37.954 & 39.639 & 38.823 & 34.087 & 13.126 & 11.920 & 12.566 & 13.431 & 19.175 & 15.424 & 18.136 & 6.325 \\
EXO_2 & 18.541 & 16.731 & 31.613 & 37.592 & 32.809 & 27.916 & 10.890 & 9.408 & 10.547 & 10.146 & 15.252 & 14.263 & 14.011 & 6.604 \\
EXO_3 & 16.941 & 16.975 & 25.398 & 35.276 & 18.794 & 24.157 & 9.794 & 6.702 & 7.770 & 9.223 & 10.475 & 11.567 & 11.966 & 5.594 \\
EXO_4 & 14.612 & 16.090 & 23.161 & 32.635 & 11.784 & 23.458 & 7.496 & 6.932 & 4.757 & 4.998 & 8.450 & 7.893 & 9.377 & 6.030 \\
EXO_best & 23.499 & 19.061 & 39.327 & 38.975 & 40.047 & 35.913 & 16.611 & 12.153 & 13.425 & 13.474 & 21.762 & 15.062 & 20.886 & 5.315 \\
GA & 31.674 & 23.375 & 45.669 & 40.149 & 48.228 & 34.372 & 22.477 & 12.788 & 23.612 & 14.104 & 30.875 & 16.530 & 28.322 & 8.458 \\
\hline
\end{tabular}

\section{RMSE}

$\left(\mathbf{W} / m^{2}\right)$

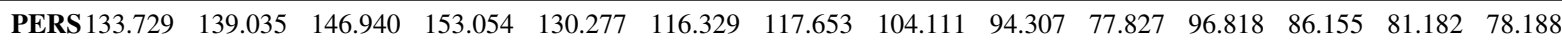

$\begin{array}{lllllllllllllll}\text { ENDO 119.030 } & 120.593 & 117.536 & 105.578 & 116.533 & 89.814 & 110.867 & 98.582 & 91.397 & 74.318 & 90.573 & 82.046 & 76.159 & 75.603\end{array}$

$\begin{array}{lllllllllllllll}\text { ANN113.870 } & 115.160 & 111.891 & 105.503 & 98.458 & 84.004 & 110.351 & 97.569 & 92.484 & 73.801 & 90.672 & 78.833 & 76.830 & 74.089\end{array}$

$\begin{array}{llllllllllllllll}\text { SVR 113.618 } & 116.925 & 107.771 & 101.380 & 98.800 & 81.814 & 112.391 & 100.384 & 89.895 & 71.843 & 93.685 & 79.623 & 77.976 & 73.741\end{array}$

$\begin{array}{lllllllllllllll}\text { EXO_1111.553 } & 112.381 & 91.170 & 92.385 & 79.700 & 76.676 & 102.210 & 91.701 & 82.456 & 67.375 & 78.254 & 72.866 & 66.459 & 73.242\end{array}$

$\begin{array}{lllllllllllllll}\text { EXO_2 108.935 } & 115.773 & 100.487 & 95.518 & 87.535 & 83.855 & 104.842 & 94.316 & 84.360 & 69.931 & 82.051 & 73.866 & 69.807 & 73.024\end{array}$

$\begin{array}{lllllllllllllll}\text { EXO_3 111.075 } & 115.434 & 109.621 & 99.063 & 105.794 & 88.228 & 106.130 & 97.134 & 86.979 & 70.650 & 86.677 & 76.189 & 71.467 & 73.814\end{array}$

$\begin{array}{llllllllllllllll}\text { EXO_4114.189 } & 116.664 & 112.907 & 103.105 & 114.926 & 89.041 & 108.834 & 96.894 & 89.821 & 73.938 & 88.638 & 79.355 & 73.570 & 73.473\end{array}$

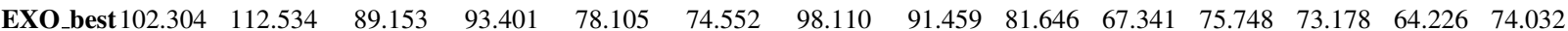
$\begin{array}{llllllllllllllll}\text { GA } 91.372 & 106.536 & 79.834 & 91.605 & 67.447 & 76.345 & 91.209 & 90.797 & 72.039 & 66.850 & 66.926 & 71.913 & 58.189 & 71.575\end{array}$

Table .6: Same as in Table .3, but for 1h-ahead horizon on a biannual basis.

\begin{tabular}{|c|c|c|c|c|c|c|c|c|c|c|c|c|c|c|}
\hline \multirow[t]{2}{*}{ s $(\%)$} & \multicolumn{2}{|c|}{ Torrey Pines } & \multicolumn{2}{|c|}{ De Laveaga } & \multicolumn{2}{|c|}{ Windsor } & \multicolumn{2}{|c|}{ McArthur } & \multicolumn{2}{|c|}{ Davis } & \multicolumn{2}{|c|}{ Merced } & \multicolumn{2}{|c|}{ Parlier } \\
\hline & Val & Test & Val & Test & Val & Test & Val & Test & Val & Test & Val & Test & Val & Test \\
\hline ENDO & 1.551 & 1.780 & 4.780 & 5.277 & 4.295 & 4.479 & 2.001 & 2.419 & 1.017 & 1.156 & 2.189 & 1.785 & 1.695 & 1.186 \\
\hline ANN & 7.154 & 5.526 & 8.324 & 9.263 & 13.832 & 14.262 & 3.045 & 4.460 & 1.794 & 1.422 & 3.781 & 4.610 & 2.639 & -0.484 \\
\hline SVR & 7.188 & 5.255 & 8.911 & 9.385 & 12.157 & 14.610 & 2.996 & 3.223 & 1.590 & 1.115 & 2.954 & 2.814 & 2.370 & 1.298 \\
\hline EXO-Lag(1) & 5.885 & 4.730 & 8.854 & 9.540 & 15.565 & 12.186 & 9.333 & 9.169 & 7.475 & 2.980 & 9.170 & 8.769 & 6.004 & 1.608 \\
\hline EXO-Lag(2) & 4.571 & 5.832 & 10.625 & 10.443 & 14.593 & 10.464 & 5.141 & 5.535 & 2.875 & 2.410 & 5.803 & 5.561 & 4.783 & 1.804 \\
\hline EXO-Lag(3) & 4.834 & 5.105 & 8.996 & 8.126 & 10.868 & 6.646 & 4.257 & 5.105 & 2.317 & 1.784 & 3.972 & 3.512 & 4.102 & 2.009 \\
\hline EXO-Lag(4) & 5.000 & 4.059 & 6.642 & 5.783 & 8.245 & 7.135 & 3.280 & 4.000 & 1.103 & 1.186 & 2.895 & 2.568 & 2.313 & 1.914 \\
\hline EXO-BestLag 1 & 14.638 & 12.485 & 17.429 & 17.258 & 22.412 & 19.333 & 11.862 & 12.836 & 8.940 & 5.446 & 12.683 & 12.389 & 9.342 & 3.849 \\
\hline GA & 18.804 & 15.908 & 22.414 & 20.968 & 27.598 & 24.595 & 17.414 & 15.471 & 17.784 & 11.238 & 16.969 & 13.408 & 16.261 & 11.301 \\
\hline \multicolumn{15}{|l|}{$\begin{array}{r}\text { RMSE } \\
\left(\mathbf{W} / m^{2}\right)\end{array}$} \\
\hline PERS 8 & 82.695 & 82.680 & 80.997 & 85.960 & 77.045 & 72.112 & 81.603 & 78.115 & 66.283 & 57.200 & 68.124 & 72.160 & 58.727 & 56.299 \\
\hline ENDO 8 & 81.412 & 81.208 & 77.125 & 81.424 & 73.736 & 68.882 & 79.970 & 76.226 & 65.609 & 56.539 & 66.633 & 70.872 & 57.732 & 55.631 \\
\hline ANN 7 & 76.779 & 78.111 & 74.255 & 77.998 & 66.388 & 61.827 & 79.118 & 74.632 & 65.093 & 56.387 & 65.548 & 68.834 & 57.178 & 56.571 \\
\hline SVR 7 & 76.751 & 78.335 & 73.779 & 77.893 & 67.679 & 61.576 & 79.158 & 75.598 & 65.229 & 56.563 & 66.112 & 70.130 & 57.336 & 55.568 \\
\hline EXO-Lag(1) 7 & 77.828 & 78.769 & 73.825 & 77.759 & 65.053 & 63.324 & 73.986 & 70.953 & 61.328 & 55.496 & 61.877 & 65.832 & 55.201 & 55.393 \\
\hline EXO-Lag(2) 7 & 78.915 & 77.858 & 72.391 & 76.983 & 65.802 & 64.566 & 77.408 & 73.792 & 64.377 & 55.822 & 64.171 & 68.147 & 55.919 & 55.283 \\
\hline EXO-Lag(3) 7 & 78.697 & 78.459 & 73.710 & 78.975 & 68.671 & 67.319 & 78.129 & 74.128 & 64.747 & 56.180 & 65.418 & 69.626 & 56.318 & 55.167 \\
\hline EXO-Lag(4) 7 & 78.561 & 79.323 & 75.617 & 80.989 & 70.692 & 66.966 & 78.926 & 74.991 & 65.552 & 56.522 & 66.152 & 70.307 & 57.369 & 55.221 \\
\hline EXO-BestLag 7 & 70.590 & 72.357 & 66.880 & 71.125 & 59.778 & 58.170 & 71.923 & 68.089 & 60.357 & 54.085 & 59.484 & 63.220 & 53.241 & 54.131 \\
\hline GA 6 & 67.145 & 69.527 & 62.842 & 67.936 & 55.782 & 54.376 & 67.393 & 66.030 & 54.495 & 50.772 & 56.564 & 62.485 & 49.178 & 49.936 \\
\hline
\end{tabular}


Table .7: Same as in Table .3, but for $2 \mathrm{~h}$-ahead horizon on a biannual basis.

\begin{tabular}{|c|c|c|c|c|c|c|c|c|c|c|c|c|c|c|}
\hline \multirow[t]{2}{*}{ s $(\%)$} & \multicolumn{2}{|c|}{ Torrey Pines } & \multicolumn{2}{|c|}{ De Laveaga } & \multicolumn{2}{|c|}{ Windsor } & \multicolumn{2}{|c|}{ McArthur } & \multicolumn{2}{|c|}{ Davis } & \multicolumn{2}{|c|}{ Merced } & \multicolumn{2}{|c|}{ Parlier } \\
\hline & Val & Test & Val & Test & Val & Test & Val & Test & Val & Test & Val & Test & Val & Test \\
\hline ENDO & 5.509 & 6.281 & 15.357 & 17.872 & 9.116 & 9.188 & 3.693 & 4.421 & 2.078 & 2.441 & 3.976 & 2.526 & 4.167 & 2.419 \\
\hline $\mathbf{A N N}$ & 11.318 & 11.126 & 17.283 & 20.589 & 21.353 & 23.975 & 6.200 & 8.176 & 3.964 & 4.664 & 7.220 & 5.769 & 7.677 & 2.564 \\
\hline SVR & 11.673 & 12.539 & 18.061 & 20.635 & 21.175 & 24.749 & 4.448 & 5.898 & 4.175 & 2.124 & 5.826 & 2.606 & 4.859 & 1.586 \\
\hline EXO-Lag(1) & 11.278 & 11.023 & 27.295 & 25.640 & 28.761 & 23.166 & 12.137 & 10.862 & 8.097 & 6.751 & 14.295 & 13.520 & 10.806 & 4.147 \\
\hline EXO-Lag(2) & 10.357 & 12.144 & 23.491 & 23.089 & 23.250 & 17.592 & 9.033 & 8.954 & 6.591 & 4.726 & 10.693 & 10.654 & 11.602 & 6.112 \\
\hline EXO-Lag3) & 11.416 & 11.176 & 21.044 & 21.743 & 17.779 & 12.272 & 7.918 & 8.821 & 3.604 & 3.351 & 7.483 & 5.649 & 6.320 & 4.403 \\
\hline EXO-Lag(4) & 10.079 & 9.744 & 18.385 & 20.308 & 11.558 & 11.074 & 5.322 & 5.431 & 2.339 & 2.575 & 5.166 & 3.840 & 5.465 & 2.627 \\
\hline EXO-BestLag & 20.634 & 17.734 & 28.076 & 26.136 & 32.408 & 27.671 & 15.408 & 16.029 & 11.785 & 9.141 & 16.942 & 15.051 & 15.065 & 6.687 \\
\hline GA & 26.253 & 18.825 & 33.054 & 30.193 & 38.931 & 32.675 & 22.579 & 16.079 & 21.150 & 14.694 & 21.875 & 16.082 & 20.712 & 8.185 \\
\hline \multicolumn{15}{|l|}{$\begin{array}{r}\text { RMSE } \\
\left(\mathbf{W} / m^{2}\right)\end{array}$} \\
\hline PERS1 & 116.385 & 120.273 & 121.470 & 128.025 & 111.829 & 101.584 & 105.982 & 98.573 & 85.274 & 70.816 & 87.340 & 85.355 & 75.313 & 72.459 \\
\hline ENDO1 & 109.973 & 112.718 & 102.817 & 105.145 & 101.635 & 92.250 & 102.068 & 94.215 & 83.502 & 69.087 & 83.867 & 83.199 & 72.175 & 70.707 \\
\hline ANN1 & 103.212 & 106.891 & 100.477 & 101.666 & 87.951 & 77.229 & 99.411 & 90.514 & 81.893 & 67.514 & 81.034 & 80.431 & 69.531 & 70.601 \\
\hline SVR 1 & 102.799 & 105.192 & 99.531 & 101.607 & 88.150 & 76.443 & 101.268 & 92.759 & 81.714 & 69.312 & 82.252 & 83.130 & 71.654 & 71.310 \\
\hline EXO-Lag(1)1 & 103.259 & 107.015 & 88.315 & 95.199 & 79.666 & 78.050 & 93.119 & 87.866 & 78.369 & 66.035 & 74.855 & 73.815 & 67.175 & 69.454 \\
\hline EXO-Lag(2)1 & 104.331 & 105.667 & 92.936 & 98.465 & 85.829 & 83.713 & 96.409 & 89.747 & 79.653 & 67.469 & 78.001 & 76.261 & 66.575 & 68.031 \\
\hline EXO-Lag3) 1 & 103.098 & 106.831 & 95.908 & 100.189 & 91.947 & 89.117 & 97.590 & 89.878 & 82.200 & 68.443 & 80.804 & 80.533 & 70.553 & 69.269 \\
\hline EXO-Lag(4) 1 & 104.654 & 108.553 & 99.138 & 102.025 & 98.905 & 90.334 & 100.342 & 93.219 & 83.279 & 68.993 & 82.827 & 82.078 & 71.198 & 70.556 \\
\hline EXO-BestLag & 92.370 & 98.943 & 87.366 & 94.564 & 75.588 & 73.475 & 89.652 & 82.772 & 75.224 & 64.343 & 72.543 & 72.508 & 63.968 & 67.614 \\
\hline GA & 85.830 & 97.631 & 81.320 & 89.371 & 68.293 & 68.391 & 82.052 & 82.723 & 67.238 & 60.410 & 68.234 & 71.628 & 59.714 & 66.528 \\
\hline
\end{tabular}

Table .8: Same as in Table .3, but for 3h-ahead horizon on a biannual basis.

\begin{tabular}{|c|c|c|c|c|c|c|c|c|c|c|c|c|c|c|}
\hline \multirow[t]{2}{*}{$\mathrm{s}(\%)$} & \multicolumn{2}{|c|}{ Torrey Pines } & \multicolumn{2}{|c|}{ De Laveaga } & \multicolumn{2}{|c|}{ Windsor } & \multicolumn{2}{|c|}{ McArthur } & \multicolumn{2}{|c|}{ Davis } & \multicolumn{2}{|c|}{ Merced } & \multicolumn{2}{|c|}{ Parlier } \\
\hline & Val & Test & Val & Test & Val & Test & Val & Test & Val & Test & Val & Test & Val & Test \\
\hline ENDO & 9.566 & 10.914 & 24.253 & 31.262 & 17.582 & 18.458 & 5.079 & 5.893 & 3.220 & 3.684 & 5.368 & 1.675 & 6.136 & 3.183 \\
\hline ANN & 14.514 & 16.332 & 24.620 & 28.689 & 27.301 & 27.294 & 8.400 & 7.940 & 6.028 & 5.599 & 8.277 & 2.070 & 7.295 & 2.334 \\
\hline SVR & 13.923 & 15.354 & 28.018 & 31.902 & 26.573 & 30.475 & 5.549 & 7.104 & 5.603 & 6.375 & 8.558 & -1.929 & 6.586 & 0.369 \\
\hline EXO-Lag(1) & 14.636 & 16.288 & 36.925 & 35.821 & 37.830 & 30.298 & 12.750 & 12.344 & 9.929 & 6.137 & 17.939 & 15.391 & 15.670 & 9.520 \\
\hline EXO-Lag(2) & 15.285 & 16.066 & 33.380 & 37.782 & 32.104 & 27.412 & 12.585 & 11.964 & 6.209 & 6.005 & 16.218 & 11.479 & 12.339 & 5.038 \\
\hline EXO-Lag3) & 15.066 & 15.335 & 31.403 & 35.864 & 25.792 & 24.022 & 10.361 & 8.426 & 4.284 & 5.068 & 11.872 & 8.497 & 8.937 & 6.297 \\
\hline EXO-Lag(4) & 14.325 & 15.206 & 25.703 & 31.276 & 19.547 & 20.705 & 6.146 & 5.654 & 2.774 & 3.216 & 6.559 & 3.993 & 7.784 & 4.477 \\
\hline EXO-BestLag & 20.693 & 19.276 & 36.697 & 35.806 & 39.953 & 35.403 & 15.177 & 12.868 & 11.350 & 7.216 & 20.907 & 16.169 & 18.396 & 9.023 \\
\hline GA & 30.222 & 24.760 & 42.148 & 41.180 & 45.478 & 37.607 & 22.659 & 17.417 & 21.450 & 12.823 & 24.078 & 16.045 & 25.452 & 9.879 \\
\hline \multicolumn{15}{|l|}{$\begin{array}{c}\text { RMSE } \\
\left(\mathbf{W} / m^{2}\right)\end{array}$} \\
\hline PERS1 & 133.729 & 139.035 & 146.940 & 153.054 & 130.277 & 116.329 & 117.653 & 104.111 & 94.307 & 77.827 & 96.818 & 86.155 & 81.182 & 78.188 \\
\hline ENDO1 & 20.937 & 123.860 & 111.303 & 105.206 & 107.371 & 94.858 & 111.678 & 97.976 & 91.270 & 74.960 & 91.621 & 84.712 & 76.201 & 75.699 \\
\hline ANN1 & 14.320 & 116.328 & 110.763 & 109.144 & 94.710 & 84.578 & 107.771 & 95.845 & 88.622 & 73.470 & 88.804 & 84.371 & 75.260 & 76.362 \\
\hline SVR 1 & 15.111 & 117.688 & 105.771 & 104.227 & 95.659 & 80.878 & 111.125 & 96.716 & 89.022 & 72.866 & 88.533 & 87.816 & 75.835 & 77.899 \\
\hline EXO-Lag(1)1 & 14.157 & 116.389 & 92.683 & 98.228 & 80.994 & 81.084 & 102.653 & 91.260 & 84.943 & 73.051 & 79.450 & 72.894 & 68.461 & 70.745 \\
\hline EXO-Lag(2) 1 & 13.289 & 116.698 & 97.891 & 95.227 & 88.453 & 84.441 & 102.847 & 91.656 & 88.451 & 73.154 & 81.116 & 76.265 & 71.165 & 74.249 \\
\hline EXO-Lag3) 1 & 13.582 & 117.715 & 100.796 & 98.162 & 96.676 & 88.384 & 105.464 & 95.339 & 90.266 & 73.883 & 85.324 & 78.834 & 73.927 & 73.264 \\
\hline EXO-Lag(4) 1 & 14.573 & 117.894 & 109.172 & 105.184 & 104.812 & 92.243 & 110.422 & 98.224 & 91.691 & 75.324 & 90.468 & 82.715 & 74.862 & 74.687 \\
\hline EXO-BestLag1 & 106.056 & 112.235 & 93.017 & 98.252 & 78.228 & 75.146 & 99.798 & 90.715 & 83.603 & 72.212 & 76.577 & 72.225 & 66.248 & 71.133 \\
\hline GA & 93.313 & 104.609 & 85.008 & 90.027 & 71.029 & 72.581 & 90.994 & 85.978 & 74.078 & 67.847 & 73.506 & 72.331 & 60.520 & 70.464 \\
\hline
\end{tabular}


Table .9: Same as in Table .3, but for 1h-ahead horizon on an annual basis

\begin{tabular}{|c|c|c|c|c|c|c|c|c|c|c|c|c|c|c|}
\hline \multirow[t]{2}{*}{ s $(\%)$} & \multicolumn{2}{|c|}{ Torrey Pines } & \multicolumn{2}{|c|}{ De Laveaga } & \multicolumn{2}{|c|}{ Windsor } & \multicolumn{2}{|c|}{ McArthur } & \multicolumn{2}{|c|}{ Davis } & \multicolumn{2}{|c|}{ Merced } & \multicolumn{2}{|c|}{ Parlier } \\
\hline & Val & Test & Val & Test & Val & Test & Val & Test & Val & Test & Val & Test & Val & Test \\
\hline ENDO & 1.535 & 1.734 & 2.121 & 1.896 & 2.337 & 2.480 & 1.754 & 1.800 & 0.731 & 0.804 & 1.415 & 1.289 & 1.006 & 0.904 \\
\hline ANN & 5.748 & 6.387 & 8.423 & 10.223 & 12.556 & 12.833 & 5.346 & 4.658 & 3.035 & 3.596 & 3.675 & 4.265 & 3.912 & 3.097 \\
\hline SVR & 6.064 & 5.355 & 8.240 & 12.067 & 13.175 & 12.722 & 3.396 & 3.767 & 2.942 & 1.222 & 2.584 & 0.667 & 2.160 & 2.272 \\
\hline EXO-Lag(1) & 4.545 & 4.738 & 10.006 & 9.586 & 15.659 & 14.451 & 8.725 & 9.147 & 7.495 & 6.056 & 9.439 & 9.865 & 5.977 & 3.823 \\
\hline EXO-Lag(2) & 3.703 & 5.672 & 10.552 & 11.612 & 12.815 & 11.518 & 5.025 & 4.621 & 2.942 & 4.446 & 4.857 & 4.666 & 3.412 & 3.516 \\
\hline EXO-Lag3) & 3.746 & 4.442 & 8.917 & 8.909 & 7.957 & 5.721 & 3.408 & 3.308 & 1.127 & 1.475 & 3.567 & 2.609 & 2.521 & 2.004 \\
\hline EXO-Lag(4) & 4.106 & 3.791 & 6.292 & 6.058 & 6.386 & 4.997 & 2.774 & 3.096 & 0.889 & 1.217 & 1.850 & 1.423 & 1.140 & 0.627 \\
\hline EXO-BestLag & 12.394 & 9.611 & 14.064 & 16.127 & 21.782 & 23.949 & 11.860 & 14.042 & 13.587 & 9.063 & 12.376 & 14.155 & 8.559 & 6.187 \\
\hline GA & 18.028 & 16.044 & 20.900 & 21.495 & 25.045 & 25.161 & 16.175 & 14.752 & 16.398 & 10.434 & 16.144 & 15.641 & 15.699 & 14.302 \\
\hline \multicolumn{15}{|l|}{$\begin{array}{r}\text { RMSE } \\
\left(\mathbf{W} / m^{2}\right)\end{array}$} \\
\hline PERS & 82.695 & 82.680 & 80.997 & 85.960 & 77.045 & 72.112 & 81.603 & 78.115 & 66.283 & 57.200 & 68.124 & 72.160 & 58.727 & 56.299 \\
\hline ENDO & 81.426 & 81.246 & 79.279 & 84.330 & 75.244 & 70.323 & 80.171 & 76.709 & 65.798 & 56.740 & 67.161 & 71.230 & 58.136 & 55.790 \\
\hline $\mathbf{A N N}$ & 77.942 & 77.398 & 74.174 & 77.173 & 67.371 & 62.857 & 77.240 & 74.477 & 64.271 & 55.143 & 65.621 & 69.083 & 56.430 & 54.555 \\
\hline SVR & 77.680 & 78.252 & 74.323 & 75.587 & 66.895 & 62.937 & 78.832 & 75.173 & 64.333 & 56.501 & 66.364 & 71.678 & 57.459 & 55.019 \\
\hline EXO-Lag(1) & 78.936 & 78.762 & 72.892 & 77.720 & 64.980 & 61.691 & 74.483 & 70.970 & 61.315 & 53.736 & 61.694 & 65.041 & 55.217 & 54.146 \\
\hline EXO-Lag(2) & 79.633 & 77.990 & 72.450 & 75.979 & 67.172 & 63.806 & 77.502 & 74.506 & 64.332 & 54.657 & 64.816 & 68.793 & 56.724 & 54.319 \\
\hline EXO-Lag3) & 79.597 & 79.007 & 73.774 & 78.302 & 70.915 & 67.986 & 78.821 & 75.531 & 65.536 & 56.357 & 65.695 & 70.277 & 57.247 & 55.170 \\
\hline EXO-Lag(4) & 79.300 & 79.545 & 75.901 & 80.753 & 72.125 & 68.508 & 79.339 & 75.697 & 65.694 & 56.504 & 66.864 & 71.133 & 58.058 & 55.946 \\
\hline EXO-BestLag & 72.445 & 74.733 & 69.605 & 72.097 & 60.263 & 54.842 & 71.925 & 67.147 & 57.277 & 52.016 & 59.693 & 61.946 & 53.701 & 52.815 \\
\hline GA & 67.787 & 69.415 & 64.068 & 67.483 & 57.749 & 53.968 & 68.404 & 66.592 & 55.414 & 51.232 & 57.127 & 60.874 & 49.508 & 48.247 \\
\hline
\end{tabular}

Table .10: Same as in Table .3, but for $2 \mathrm{~h}$-ahead horizon on an annual basis.

\begin{tabular}{|c|c|c|c|c|c|c|c|c|c|c|c|c|c|c|}
\hline \multirow[t]{2}{*}{ s $(\%)$} & \multicolumn{2}{|c|}{ Torrey Pines } & \multicolumn{2}{|c|}{ De Laveaga } & \multicolumn{2}{|c|}{ Windsor } & \multicolumn{2}{|c|}{ McArthur } & \multicolumn{2}{|c|}{ Davis } & \multicolumn{2}{|c|}{ Merced } & \multicolumn{2}{|c|}{ Parlier } \\
\hline & Val & Test & Val & Test & Val & Test & Val & Test & Val & Test & Val & Test & Val & Test \\
\hline ENDO & 5.093 & 5.918 & 8.770 & 8.682 & 4.468 & 4.634 & 3.284 & 3.129 & 1.668 & 1.884 & 2.903 & 2.275 & 2.659 & 2.087 \\
\hline ANN & 10.546 & 12.161 & 15.205 & 18.371 & 21.160 & 20.312 & 8.302 & 7.004 & 6.532 & 5.322 & 6.551 & 6.012 & 6.773 & 4.022 \\
\hline SVR & 10.780 & 12.415 & 19.158 & 20.858 & 21.253 & 20.045 & 5.544 & 5.122 & 4.655 & 1.471 & 4.406 & 5.257 & 3.929 & 1.456 \\
\hline EXO-Lag(1) & 8.600 & 10.413 & 26.981 & 27.535 & 26.075 & 24.836 & 11.738 & 10.337 & 9.808 & 12.069 & 12.835 & 12.131 & 10.772 & 8.491 \\
\hline EXO-Lag(2) & 7.857 & 9.314 & 24.238 & 24.211 & 20.317 & 15.545 & 7.867 & 7.227 & 5.291 & 7.001 & 9.796 & 8.129 & 8.570 & 6.198 \\
\hline EXO-Lag3) & 10.046 & 9.805 & 17.052 & 19.984 & 16.199 & 12.538 & 8.507 & 7.632 & 1.889 & 2.084 & 5.407 & 5.414 & 5.767 & 2.103 \\
\hline EXO-Lag(4) & 9.113 & 8.973 & 11.869 & 12.457 & 8.728 & 7.526 & 4.058 & 5.063 & 2.482 & 1.664 & 3.012 & 2.267 & 3.835 & 2.391 \\
\hline EXO-BestLag & 18.035 & 15.902 & 29.117 & 29.796 & 31.335 & 29.762 & 13.472 & 12.206 & 12.709 & 13.218 & 15.074 & 12.199 & 13.167 & 8.918 \\
\hline GA & 24.448 & 20.084 & 31.402 & 31.259 & 35.322 & 32.455 & 19.343 & 14.778 & 17.374 & 13.532 & 18.594 & 15.408 & 17.469 & 10.428 \\
\hline \multicolumn{15}{|l|}{$\begin{array}{r}\text { RMSE } \\
\left(\mathbf{W} / m^{2}\right)\end{array}$} \\
\hline PERS1 & 16.385 & 120.273 & 121.470 & 128.025 & 111.829 & 101.584 & 105.982 & 98.573 & 85.274 & 70.816 & 87.340 & 85.355 & 75.313 & 72.459 \\
\hline ENDO1 & 10.457 & 113.155 & 110.817 & 116.910 & 106.833 & 96.876 & 102.502 & 95.488 & 83.851 & 69.482 & 84.804 & 83.413 & 73.311 & 70.947 \\
\hline ANN1 & 04.111 & 105.646 & 103.001 & 104.505 & 88.166 & 80.950 & 97.184 & 91.669 & 79.704 & 67.047 & 81.618 & 80.224 & 70.212 & 69.545 \\
\hline SVR 1 & 03.839 & 105.341 & 98.200 & 101.322 & 88.062 & 81.222 & 100.107 & 93.524 & 81.304 & 69.774 & 83.491 & 80.868 & 72.354 & 71.404 \\
\hline EXO-Lag(1)1 & 06.375 & 107.748 & 88.696 & 92.773 & 82.670 & 76.355 & 93.542 & 88.384 & 76.910 & 62.269 & 76.130 & 75.000 & 67.200 & 66.307 \\
\hline EXO-Lag(2) 1 & 07.240 & 109.071 & 92.028 & 97.029 & 89.109 & 85.792 & 97.644 & 91.449 & 80.762 & 65.858 & 78.784 & 78.417 & 68.859 & 67.968 \\
\hline EXO-Lag3) 1 & 04.693 & 108.479 & 100.757 & 102.440 & 93.714 & 88.847 & 96.967 & 91.049 & 83.663 & 69.341 & 82.617 & 80.734 & 70.970 & 70.935 \\
\hline EXO-Lag(4)1 & 05.778 & 109.481 & 107.054 & 112.077 & 102.069 & 93.939 & 101.682 & 93.582 & 83.157 & 69.638 & 84.709 & 83.420 & 72.425 & 70.727 \\
\hline EXO-BestLag & 95.395 & 101.146 & 86.102 & 89.879 & 76.788 & 71.350 & 91.704 & 86.541 & 74.436 & 61.456 & 74.174 & 74.942 & 65.397 & 65.998 \\
\hline GA & 87.931 & 96.117 & 83.326 & 88.005 & 72.329 & 68.615 & 85.482 & 84.006 & 70.458 & 61.233 & 71.100 & 72.204 & 62.157 & 64.903 \\
\hline
\end{tabular}


Table .11: Same as in Table .3, but for 3h-ahead horizon on an annual basis.

\begin{tabular}{|c|c|c|c|c|c|c|c|c|c|c|c|c|c|c|}
\hline \multirow[t]{2}{*}{ s $(\%)$} & \multicolumn{2}{|c|}{ Torrey Pines } & \multicolumn{2}{|c|}{ De Laveaga } & \multicolumn{2}{|c|}{ Windsor } & \multicolumn{2}{|c|}{ McArthur } & \multicolumn{2}{|c|}{ Davis } & \multicolumn{2}{|c|}{ Merced } & \multicolumn{2}{|c|}{ Parlier } \\
\hline & Val & Test & Val & Test & Val & Test & Val & Test & Val & Test & Val & Test & Val & Test \\
\hline ENDO & 9.013 & 10.508 & 16.477 & 17.148 & 7.633 & 7.853 & 4.469 & 4.125 & 2.740 & 2.997 & 4.312 & 2.590 & 4.424 & 3.020 \\
\hline ANN & 13.095 & 15.540 & 23.136 & 26.614 & 26.421 & 24.349 & 9.689 & 4.704 & 6.659 & 7.645 & 7.568 & 6.063 & 8.414 & 7.061 \\
\hline SVR & 13.432 & 15.841 & 25.757 & 29.908 & 26.199 & 23.968 & 7.251 & 3.044 & 6.212 & 5.104 & 5.271 & 2.333 & 4.631 & 4.148 \\
\hline EXO-Lag(1) & 11.773 & 14.306 & 36.331 & 36.840 & 32.886 & 28.422 & 12.752 & 12.231 & 10.342 & 11.884 & 16.083 & 12.601 & 14.673 & 9.128 \\
\hline EXO-Lag(2) & 13.324 & 14.258 & 33.324 & 36.188 & 28.133 & 21.488 & 11.937 & 9.825 & 5.862 & 7.210 & 12.761 & 10.350 & 11.063 & 7.098 \\
\hline EXO-Lag3) & 14.100 & 14.704 & 30.314 & 31.284 & 20.348 & 17.700 & 10.647 & 7.526 & 4.724 & 4.708 & 9.402 & 7.350 & 9.446 & 5.894 \\
\hline EXO-Lag(4) & 12.215 & 14.168 & 20.560 & 20.827 & 12.869 & 14.236 & 6.144 & 5.481 & 3.270 & 2.526 & 5.794 & 3.152 & 5.346 & 4.121 \\
\hline EXO-BestLag & 21.057 & 19.766 & 37.059 & 37.847 & 37.200 & 33.876 & 15.016 & 13.261 & 11.601 & 13.234 & 18.302 & 12.990 & 15.034 & 6.032 \\
\hline GA & 27.471 & 22.442 & 39.897 & 39.196 & 40.747 & 37.312 & 19.012 & 15.749 & 15.992 & 12.871 & 23.010 & 14.494 & 22.758 & 8.969 \\
\hline \multicolumn{15}{|l|}{$\begin{array}{r}\text { RMSE } \\
\left(\mathbf{W} / m^{2}\right)\end{array}$} \\
\hline \multicolumn{2}{|c|}{ PERS133.729 } & 139.035 & 46.940 & 53.054 & 130.277 & & 117.653 & 104.111 & 94.307 & 77.827 & 96.818 & 86.155 & 81.182 & 78.188 \\
\hline \multicolumn{2}{|c|}{ ENDO121.676 } & 124.425 & 122.729 & 126.808 & 120.334 & 107.194 & 112.396 & 99.816 & 91.723 & 75.495 & 92.643 & 83.923 & 77.590 & 75.827 \\
\hline \multicolumn{2}{|c|}{ ANN116.217 } & 117.430 & 112.943 & 112.320 & 95.857 & 88.004 & 106.254 & 99.214 & 88.027 & 71.878 & 89.491 & 80.931 & 74.352 & 72.667 \\
\hline \multicolumn{2}{|c|}{ SVR115.767 } & 117.010 & 109.093 & 107.278 & 96.145 & 88.447 & 109.123 & 100.942 & 88.449 & 73.855 & 91.715 & 84.144 & 77.422 & 74.944 \\
\hline \multicolumn{2}{|c|}{ EXO-Lag(1)117.986 } & 119.145 & 93.554 & 96.669 & 87.434 & 83.267 & 102.651 & 91.377 & 84.554 & 68.578 & 81.247 & 75.298 & 69.270 & 71.051 \\
\hline \multicolumn{2}{|c|}{ EXO-Lag(2)115.912 } & 119.212 & 97.973 & 97.666 & 93.627 & 91.333 & 103.609 & 93.883 & 88.778 & 72.216 & 84.463 & 77.238 & 72.201 & 72.638 \\
\hline \multicolumn{2}{|c|}{ EXO-Lag3) 114.874} & 118.592 & 102.396 & 105.173 & 103.768 & 95.739 & 105.127 & 96.276 & 89.851 & 74.163 & 87.715 & 79.822 & 73.513 & 73.579 \\
\hline \multicolumn{2}{|c|}{ EXO-Lag(4)117.394 } & 119.337 & 116.729 & 121.177 & 113.511 & 99.769 & 110.424 & 98.405 & 91.223 & 75.861 & 91.209 & 83.439 & 76.842 & 74.965 \\
\hline \multicolumn{2}{|c|}{ EXO-BestLag105.571 } & 111.553 & 92.485 & 95.128 & 81.814 & 76.922 & 99.986 & 90.305 & 83.366 & 67.527 & 79.099 & 74.963 & 68.977 & 73.471 \\
\hline \multicolumn{2}{|c|}{ GA 96.993} & 107.833 & 88.315 & 93.063 & 77.193 & 72.925 & 95.285 & 87.715 & 79.226 & 67.810 & 74.541 & 73.667 & 62.706 & 71.175 \\
\hline
\end{tabular}

